\title{
Probing the Role of Carbon in the Ultraviolet Interstellar Extinction
}

\author{
Ajay Mishra and Aigen Li \\ Department of Physics and Astronomy, University of Missouri, Columbia, MO 65211, \\ USA; amishra@mail.missouri.edu, lia@missouri.edu
}

\begin{abstract}
We probe the role of carbon in the ultraviolet (UV) extinction by examining the relations between the amount of carbon required to be locked up in dust $[\mathrm{C} / \mathrm{H}]_{\text {dust }}$ with the $2175 \AA$ extinction bump and the far-UV extinction rise, based on an analysis of the extinction curves along 16 Galactic sightlines for which the gas-phase carbon abundance is known and the $2175 \AA$ extinction bump exhibits variable strengths and widths. We derive $[\mathrm{C} / \mathrm{H}]_{\text {dust }}$ from the Kramers-Kronig relation which relates the wavelength-integrated extinction to the total dust volume. This approach is less model-dependent since it does not require the knowledge of the detailed optical properties and size distribution of the dust. We also derive $[\mathrm{C} / \mathrm{H}]_{\text {dust }}$ from fitting the observed UV/optical/near-infrared extinction with a mixture of amorphous silicate and graphite. We find that the carbon depletion $[\mathrm{C} / \mathrm{H}]_{\text {dust }}$ tends to correlate with the strength of the $2175 \AA$ bump, while the abundance of silicon depleted in dust shows no correlation with the $2175 \AA$ bump. This supports graphite or polycyclic aromatic hydrocarbon (PAH) molecules as the possible carrier of the $2175 \AA$ bump. We also see that $[\mathrm{C} / \mathrm{H}]_{\text {dust }}$ shows a trend of correlating with $1 / R_{V}$, where $R_{V}$ is the total-to-selective extinction ratio, suggesting that the far-UV extinction is more likely produced by small carbon dust than by small silicate dust.
\end{abstract}

Subject headings: dust, extinction — ISM: abundances — ISM: clouds

\section{Introduction}

Dust is a ubiquitous feature of the cosmos. It has dramatic effects on the interstellar medium (ISM) by affecting the interstellar conditions, participating in the physical and chemical processes occuring in the ISM, and shaping the appearance of dusty objects as an absorber, scatterer, and emitter of electromagnetic radiation, and as an efficient catalyst for 
the formation of $\mathrm{H}_{2}$ and an agent for photoelectrically heating the interstellar gas. To understand the interaction of the solid dust particles in the space between stars (i.e., interstellar dust) with radiation and the dust-related interstellar processes, the knowledge of the dust composition and size is required.

What is interstellar dust made of? The ubiquitous detection of the $9.7 \mu \mathrm{m} \mathrm{Si-O}$ stretching and $18 \mu \mathrm{m} \mathrm{O-Si-O} \mathrm{bending} \mathrm{absorption} \mathrm{features} \mathrm{in} \mathrm{interstellar} \mathrm{regions} \mathrm{implies} \mathrm{that} \mathrm{silicate}$ must be a major component (Henning 2010). These features are broad, smooth and featureless, suggesting that interstellar silicate is amorphous (Li \& Draine 2001a, Kemper et al. 2004, Li et al. 2008). Another absorption feature ubiquitously seen in the diffuse ISM of the Milky Way and external galaxies is the $3.4 \mu \mathrm{m}$ feature, commonly attributed to the C$\mathrm{H}$ stretch in some sorts of aliphatic hydrocarbon materials (see Pendleton \& Allamandola 2002). Depending on their $\mathrm{H}$ fractions and the preparation method, the experimentallysynthesized aliphatic hydrocarbon analogues show considerable variation in the $\mathrm{C}-\mathrm{H}$ band strength (e.g., see Furton, Laiho, \& Witt 1999). Therefore, it has not been possible to use the measured $3.4 \mu \mathrm{m}$ feature to determine the abundance of aliphatic hydrocarbon material in the ISM (e.g., see Chiar et al. 2013).

One may infer the amount of silicate dust $M_{\text {sil }}$ from the interstellar $\mathrm{Si}, \mathrm{Mg}$, and Fe abundances. Let $[\mathrm{X} / \mathrm{H}]_{\mathrm{ISM}}$ be the total interstellar abundance of element $\mathrm{X}$ relative to $\mathrm{H}$, $[\mathrm{X} / \mathrm{H}]_{\text {gas }}$ be the amount of $\mathrm{X}$ in the gas phase, and $[\mathrm{X} / \mathrm{H}]_{\text {dust }}$ be the amount of $\mathrm{X}$ contained in dust. As an element will be in gas-phase and/or locked up in solid-phase, we will obviously have $[\mathrm{X} / \mathrm{H}]_{\text {dust }}=[\mathrm{X} / \mathrm{H}]_{\mathrm{ISM}}-[\mathrm{X} / \mathrm{H}]_{\text {gas }}$. Let $\mu_{\mathrm{X}}$ be the atomic weight of $\mathrm{X}\left(\mu_{\mathrm{X}}=12,16,56\right.$, 24 , and 28 for carbon, oxygen, iron, magnesium, and silicon). If we assume a stoichiometric composition of $\mathrm{Mg}_{2 \mathrm{x}} \mathrm{Fe}_{2(1-\mathrm{x})} \mathrm{SiO}_{4}$ for silicate (i.e., each silicon atom corresponds to four oxygen atoms), the interstellar silicate dust mass relative to hydrogen is

$$
M_{\mathrm{sil}} / M_{\mathrm{H}}=\mu_{\mathrm{Fe}}[\mathrm{Fe} / \mathrm{H}]_{\mathrm{dust}}+\mu_{\mathrm{Mg}}[\mathrm{Mg} / \mathrm{H}]_{\mathrm{dust}}+\mu_{\mathrm{Si}}[\mathrm{Si} / \mathrm{H}]_{\text {dust }}+4 \times \mu_{\mathrm{O}}[\mathrm{Si} / \mathrm{H}]_{\mathrm{dust}}
$$

The exact interstellar abundances for the dust-forming elements are unknown (see Snow \& Witt 1996, Sofia 2004, Li 2005, Jenkins 2009). The abundances of the Sun, the proto-Sun, unevolved early B stars, and F and G stars are often adopted as the interstellar reference abundance standards. If we adopt the proto-Sun abundances of $[\mathrm{Fe} / \mathrm{H}]_{\odot}=34.7 \pm 2.4 \mathrm{ppm}$, $[\mathrm{Mg} / \mathrm{H}]_{\odot}=41.7 \pm 1.9 \mathrm{ppm}$, and $[\mathrm{Si} / \mathrm{H}]_{\odot}=40.7 \pm 1.9 \mathrm{ppm}$ (Lodders 2003) for the interstellar reference abundances and assume that $\mathrm{Fe}, \mathrm{Mg}$ and $\mathrm{Si}$ are essentially completely locked up in dust (i.e., $[\mathrm{X} / \mathrm{H}]_{\text {dust }}=[\mathrm{X} / \mathrm{H}]_{\mathrm{ISM}}$ for $\mathrm{Fe}, \mathrm{Mg}$ and $\mathrm{Si}$ ), we obtain $M_{\text {sil }} / M_{\mathrm{H}} \approx 6.69 \times 10^{-3}$. Let $C_{\text {abs }}(9.7 \mu \mathrm{m}) / V=1.00 \times 10^{4} \mathrm{~cm}^{-1}$ be the peak absorption cross section per volume of silicate dust at $9.7 \mu \mathrm{m}$ (Draine \& Lee 1984) and $A_{V} / N_{\mathrm{H}}=5.3 \times 10^{-22} \mathrm{mag} \mathrm{cm}^{2} \mathrm{H}^{-1}$ be the visual extinction $A_{V}$ per hydrogen column $N_{\mathrm{H}}$ (Whittet 2003). One may estimate the ratio 
of the optical depth of the $9.7 \mu \mathrm{m}$ silicate absorption feature to $A_{V}$ to be

$$
\Delta \tau_{9.7} / A_{V} \approx\left[C_{\text {abs }}(9.7 \mu \mathrm{m}) / V\right] / \rho_{\text {sil }} \times\left(M_{\text {sil }} / M_{\mathrm{H}}\right) \times\left(N_{\mathrm{H}} / A_{V}\right) \times m_{\mathrm{H}} \approx 1 / 16.7,
$$

where $\rho_{\text {sil }} \approx 3.5 \mathrm{~g} \mathrm{~cm}^{-3}$ is the mass density of silicate and $m_{\mathrm{H}}=1.66 \times 10^{-24} \mathrm{~g}$ is the mass of a hydrogen atom. This is close to the mean value of $\Delta \tau_{9.7} / A_{V} \approx 1 / 18.5$ observed for the local diffuse ISM (see Draine 2003a), suggesting that the proto-Sun Fe, Mg and Si abundances are sufficient to account for the observed $9.7 \mu \mathrm{m}$ silicate absorption feature 1

However, silicate alone is not sufficient to account for the observed interstellar extinction. As shown by Purcell (1969), the Kramers-Kronig relation can be used to constrain the dust quantity in the ISM. Let $A_{\lambda}$ be the extinction at wavelength $\lambda$ and $A_{\lambda} / N_{\mathrm{H}}$ be the extinction per $\mathrm{H}$ nucleon. As shown in $\mathrm{Li}$ (2005), the Kramers-Kronig relation relates the wavelengthintegrated extinction $\left(\int_{0}^{\infty} A_{\lambda} / N_{\mathrm{H}} d \lambda\right)$ to the total volume occupied by dust per $\mathrm{H}$ nucleon $\left(V_{\text {dust }} / \mathrm{H}\right)$ through

$$
\int_{0}^{\infty} \frac{A_{\lambda}}{N_{\mathrm{H}}} d \lambda=1.086 \times 3 \pi^{2} F \frac{V_{\text {dust }}}{\mathrm{H}},
$$

where $F$ is a dimensionless factor and depends only upon the grain shape and the static (zero-frequency) dielectric constant $\varepsilon_{0}$ of the grain material (see below). The interstellar extinction per $\mathrm{H}$ nucleon $\left(A_{\lambda} / N_{\mathrm{H}}\right)$ is not known for all wavelengths, but for a limited range of wavelengths. We approximate $\int_{0}^{\infty} A_{\lambda} / N_{\mathrm{H}} d \lambda$ by $\int_{912 \AA}^{1000 \mu \mathrm{m}} A_{\lambda} / N_{\mathrm{H}} d \lambda$, with the latter derived from the silicate-graphite-PAH model of Weingartner \& Draine (2001; hereafter WD01) which closely reproduces the observed extinction from the far-UV to the near-IR and up to $\lambda \approx 30 \mu \mathrm{m}$ (see Figure 16 of Li \& Draine 2001b). The WD01 model gives a reasonably accurate estimate for the extinction at $\lambda>30 \mu \mathrm{m}$ which is observationally unknown since this model closely fits the observed dust thermal emission from the near-IR to submillimeter 2

${ }^{1}$ If we adopt the abundances of unevolved early B-type stars $\left([\mathrm{Fe} / \mathrm{H}]_{\star}=27.5 \pm 2.5 \mathrm{ppm},[\mathrm{Mg} / \mathrm{H}]_{\star}=\right.$ $36.3 \pm 4.2 \mathrm{ppm}$, and $[\mathrm{Si} / \mathrm{H}]_{\star}=31.6 \pm 1.5 \mathrm{ppm}$; Przybilla et al. 2008) for the interstellar reference abundances, we obtain $\Delta \tau_{9.7} / A_{V} \approx 1 / 21.0$. This is somewhat lower than the observed value of $\Delta \tau_{9.7} / A_{V} \approx 1 / 18.5$. The abundances of the silicate-forming elements of the Sun $\left([\mathrm{Fe} / \mathrm{H}]_{\odot}=31.6 \pm 2.9 \mathrm{ppm},[\mathrm{Mg} / \mathrm{H}]_{\odot}=39.8 \pm 3.7 \mathrm{ppm}\right.$, and $[\mathrm{Si} / \mathrm{H}]_{\odot}=32.4 \pm 2.2 \mathrm{ppm}$; Asplund et al. 2009) and of young $\mathrm{F}$ and $\mathrm{G}$ stars $\left([\mathrm{Fe} / \mathrm{H}]_{\star}=28.2 \pm 7.8 \mathrm{ppm}\right.$, $[\mathrm{Mg} / \mathrm{H}]_{\star}=42.7 \pm 16.7 \mathrm{ppm}$, and $[\mathrm{Si} / \mathrm{H}]_{\star}=39.8 \pm 12.8 \mathrm{ppm}$; Sofia \& Meyer 2001) are somewhat intermediate between that of the proto-Sun and early B stars, except the magnesium abundance of young $\mathrm{F}$ and $\mathrm{G}$ stars is a little bit higher than that of the proto-Sun and early B stars (but we note that the abundances of young F and G stars have large uncertainties; see Sofia \& Meyer 2001).

${ }^{2}$ The WD01 model reproduces the observed UV/optical extinction as well as the albedo (see Figure 15 of Li \& Draine 2001b). This implies that the WD01 model gives a reasonably accurate description on how interstellar dust absorbs starlight. On the other hand, the fact that the WD01 model closely reproduces the observed IR emission indicates that this model also gives a reasonably accurate description on how interstellar dust emits in the IR and of its IR opacities. 
For the diffuse ISM, the WD01 model gives $\int_{912 \AA}^{1000 \mu \mathrm{m}} A_{\lambda} / N_{\mathrm{H}} d \lambda \approx 1.49 \times 10^{-25} \mathrm{mag} \mathrm{cm}^{3} \mathrm{H}^{-1} 3$ Since $A_{\lambda}$ is a positive number and $V_{\text {dust }} / N_{\mathrm{H}}$ is obtained by integrating $A_{\lambda} / N_{\mathrm{H}}$ over all wavelengths (see eq.33), therefore, the integration of $A_{\lambda} / N_{\mathrm{H}}$ over a finite wavelength range can be used to place a lower limit on the total amount of dust in the ISM.

If we take the dust to be spheroids with semiaxes $a, b, b$ (prolate if $a / b>1$, oblate if $a / b<1$ ), then $F$ is related to the static dielectric constant $\varepsilon_{0}$ and the "depolarization factors" $L_{a}$ and $L_{b}=\left(1-L_{a}\right) / 2$ through (Draine 2003b)

$$
F\left(a / b, \varepsilon_{0}\right) \equiv \frac{\left(\varepsilon_{0}-1\right)}{9}\left[\frac{1}{\left(\varepsilon_{0}-1\right) L_{a}+1}+\frac{2}{\left(\varepsilon_{0}-1\right) L_{b}+1}\right],
$$

where for prolates

$$
L_{a}=\frac{1-e^{2}}{e^{2}}\left[\frac{1}{2 e} \ln \left(\frac{1+e}{1-e}\right)-1\right],
$$

and for oblates

$$
L_{a}=\frac{1+e^{2}}{e^{2}}\left[1-\frac{1}{e} \arctan (e)\right],
$$

where

$$
e \equiv\left|1-(b / a)^{2}\right|^{1 / 2}
$$

In Figure 1 we show the $F$ factors for silicate, $\mathrm{Fe}_{2} \mathrm{O}_{3}$, amorphous carbon, and graphite. These dust species are often invoked to account for the interstellar extinction. They also represent materials from being dielectric (silicate with $\varepsilon_{0} \approx 6.4,4 \mathrm{Fe}_{2} \mathrm{O}_{3}$ with $\varepsilon_{0} \approx 16$ ) to somewhat conducting (amorphous carbon with $\varepsilon_{0} \approx 160$ ) to highly conducting (graphite with $\left.\varepsilon_{0} \rightarrow \infty\right)$. For silicate spheres we have $F \approx 0.64$. For modestly elongated or flattened silicate spheroids, $F$ does not exceed unity: $F \approx 0.73$ for $a / b=3$ (Greenberg \& Li 1996) 5

\footnotetext{
${ }^{3}$ In the far-IR, the extinction rapidly declines with wavelength (e.g., $A_{\lambda} \propto \lambda^{-2}$, see Table 6 of Li \& Draine 2001b) and therefore, the contribution of the extinction at $\lambda>1000 \mu \mathrm{m}$ to the extinction integration is negligible. Indeed, the wavelength-integrated extinction $\int A_{\lambda} / N_{\mathrm{H}} d \lambda$ only increases $\sim 1.6 \%$ by extending the upper integral limit from $\lambda=1000 \mu \mathrm{m}$ to $10,000 \mu \mathrm{m}$.

${ }^{4}$ Davis et al. (1988) measured the temperature dependence of the static dielectric constant $\varepsilon_{0}$ of naturally occurring monocrystalline forsterite $\mathrm{Mg}_{1.86} \mathrm{Fe}_{0.14} \mathrm{SiO}_{4}$. They found that $\varepsilon_{0}$ depends linearly upon the temperature: $\varepsilon_{0} \approx 6.3+0.0064 \times T$. For typical dust temperatures of $T \approx 20 \mathrm{~K}$ in the ISM (Li \& Draine $2001 \mathrm{~b}$ ), one obtains $\varepsilon_{0} \approx 6.4$ for silicates.

${ }^{5}$ Greenberg \& Li (1996) modeled the 9.7 and $18 \mu \mathrm{m}$ silicate polarization features toward the BecklinNeugebauer (BN) object in terms of core-mantle spheroids. They found that the $a / b=3$ prolate shape provides the best fit.
} 
and $F \approx 0.68$ for $a / b=1 / 2$ (Lee \& Draine 1985).6 Assuming the proto-Sun abundances for the interstellar reference abundances, the total silicate volume per $\mathrm{H}$ nucleon is

$$
V_{\mathrm{sil}} / \mathrm{H} \approx\left(M_{\mathrm{sil}} / M_{\mathrm{H}}\right) / \rho_{\mathrm{sil}} \times m_{\mathrm{H}} \approx 3.17 \times 10^{-27} \mathrm{~cm}^{3} \mathrm{H}^{-1}
$$

With $F \approx 0.7$ for moderately elongated or flattened silicate spheroids, the wavelengthintegrated extinction arising from the silicate dust component is at most $\approx 7.14 \times 10^{-26} \mathrm{mag} \mathrm{cm}^{3} \mathrm{H}^{-1} .7$ Apparently, this is not sufficient to account for the observed lower limit of $\approx 1.49 \times 10^{-25} \mathrm{mag} \mathrm{cm}^{3} \mathrm{H}^{-1}$. Therefore, there must exist other dust component(s), with carbon dust being the most popular one 8 As a matter of fact, all modern dust models invoke some form of carbonaceous materials including graphite (Mathis et al. 1977, hereafter MRN; Draine \& Lee 1984), "organic refractory" (Li \& Greenberg 1997), and hydrogenated amorphous carbon (HAC; Duley et al. 1989, Jones et al. 2013). We note that carbon-bearing dust species have revealed their presence in the ISM through the $3.4 \mu \mathrm{m}$ aliphatic $\mathrm{C}-\mathrm{H}$ absorption feature (Pendleton \& Allamandola 2002), the "unidentified" infrared emission (UIE) bands at 3.3, 6.2, 7.7, 8.6, 11.3 and $12.7 \mu \mathrm{m}$ (Léger \& Puget 1984, Allamandola et al. 1985), and the "extended Red emission" (ERE) attributed to the photoluminescence of some form of carbon dust (Witt \& Vijh 2004).

What role does the carbonaceous dust component play in the UV/optical extinction? as elaborated above, it must account for $\sim 52 \%$ of the total interstellar extinction. Spectroscopically, the $2175 \AA$ extinction bump, the strongest absorption feature observed in the ISM, was widely attributed to small graphite grains (Stecher \& Donn 1965, Draine \& Malhotra 1993, Mathis 1994) or polycyclic aromatic hydrocarbon (PAH) molecules (Joblin et al. 1992, Li \& Draine 2001b, Cecchi-Pestellini et al. 2008, Steglich et al. 2010, Mulas et al. 2013). It was thought that the $\pi-\pi^{*}$ transition in $s p^{2}$ hybridization of aromatic carbon causes the

\footnotetext{
${ }^{6}$ The $a / b=1 / 2$ oblate shape was shown to provide a better match than any other shapes to the $3.1 \mu \mathrm{m}$ ice polarization feature (Lee \& Draine 1985) and the BN $9.7 \mu \mathrm{m}$ silicate polarization (Hildebrand \& Dragovan 1995). Unlike Greenberg \& Li (1996), Hildebrand \& Dragovan (1995) considered bare silicate dust and only modeled the $9.7 \mu \mathrm{m}$ polarization.

${ }^{7}$ This reduces to $\approx 5.68 \times 10^{-26} \mathrm{mag} \mathrm{cm}^{3} \mathrm{H}^{-1}$ if the $\mathrm{Fe}, \mathrm{Mg}$ and $\mathrm{Si}$ abundances of unevolved early B stars (Przybilla et al. 2008) are adopted. The $V_{\text {sil }} / \mathrm{H}$ values obtained with the abundances of young $\mathrm{F}$ and $\mathrm{G}$ stars (Sofia \& Meyer 2001) or the Sun (Asplund et al. 2009) are intermediate between that of the proto-Sun and that of the early B stars.

${ }^{8}$ Other dust species such as iron and iron oxides may also be present in the ISM. However, if a substantially large fraction of the interstellar $\mathrm{Fe} / \mathrm{H}$ abundance is consumed by iron or iron oxides, the interstellar silicate grains will not be "dirty" enough to account for the observed optical/near-IR extinction (see Draine \& Lee 1984). Moreover, iron oxides have absorption peaks at $\sim 9.2,18,21$ and $30 \mu \mathrm{m}$ for hematite $\alpha$-Fe $\mathrm{O}_{3}$ and $\sim 17$ and $25 \mu \mathrm{m}$ for magnetite $\mathrm{Fe}_{3} \mathrm{O}_{4}$ which are not seen in the ISM (see Zhang, Jiang, \& Li 2009).
} 
$2175 \AA$ extinction bump. However, Parvathi et al. (2012) recently examined the correlation of the carbon depletion $[\mathrm{C} / \mathrm{H}]_{\text {dust }}$ (i.e., the number of carbon atoms [relative to $\mathrm{H}$ ] locked up in dust) with the strength of the $2175 \AA$ extinction bump for 21 Galactic lines of sight and found no correlation. This provides a severe challenge to the hypothesis of graphite or PAHs as the carriers of the $2175 \AA$ bump.

Parvathi et al. (2012) derived the dust-phase carbon abundance $[\mathrm{C} / \mathrm{H}]_{\text {dust }}$ for each sightline by subtracting the gas-phase carbon abundance $[\mathrm{C} / \mathrm{H}]_{\text {gas }}$ from the interstellar reference abundance $[\mathrm{C} / \mathrm{H}]_{\mathrm{ISM}}:[\mathrm{C} / \mathrm{H}]_{\text {dust }}=[\mathrm{C} / \mathrm{H}]_{\mathrm{ISM}}-[\mathrm{C} / \mathrm{H}]_{\text {gas }}$. The gas-phase carbon abundance $[\mathrm{C} / \mathrm{H}]_{\text {gas }}$ was determined by Sofia et al. (2011) for six translucent sightlines and by Parvathi et al. (2012) for 15 sightlines. Their analysis was based on the strong $1334 \AA$ absorption line of CII observed by the Space Telescope Imaging Spectrograph (STIS) on board the Hubble Space Telescope (HST). As described in Parvathi et al. (2012), these sightlines span a variety of Galactic disk environments which are characterized by different extinction and sample paths ranging over three orders of magnitude in $\left\langle n_{\mathrm{H}}\right\rangle$, the average density of hydrogen. They have all been observed with the Far-Ultraviolet Spectroscopic Explorer (FUSE) and had their total neutral hydrogen (i.e., $\mathrm{HI}$ and $\mathrm{H}_{2}$ ) column densities measured by Cartledge et al. (2004, 2006). Parvathi et al. (2012) took the total interstellar carbon abundance to be $[\mathrm{C} / \mathrm{H}]_{\mathrm{ISM}}=464 \mathrm{ppm}$. They assumed that the gas-phase carbon abundance toward HD $206773\left([\mathrm{C} / \mathrm{H}]_{\text {gas }} \approx 464 \pm 57 \mathrm{ppm}\right)$ represents the lower limit for the interstellar cosmic carbon abundance.

In this work we revisit the Galactic sightlines considered by Parvathi et al. (2012) to probe the role of the carbonaceous dust component in the UV extinction, with special attention paid to the $2175 \AA$ extinction bump. We take two approaches both of which differ from that of Parvathi et al. (2012). One approach is independent of any dust models: we use the Kramers-Kronig relation of Purcell (1969) to relate the wavelength-integrated extinction to the total dust volume (see eq.33) to infer the carbon depletion $[\mathrm{C} / \mathrm{H}]_{\text {dust }}$ for each sightline (see \$2). We then examine the relation between $[\mathrm{C} / \mathrm{H}]_{\text {dust }}$ and the $2175 \AA$ extinction bump strength and the far-UV extinction rise (see \$2). Another approach is to model the observed extinction curve of each sightline in terms of the silicate-graphite model (see $\$ 3$ ). Again, the required carbon depletion in graphite is compared with the $2175 \AA$ extinction bump strength and the far-UV extinction rise (see \$3). The results are discussed in $\$ 4$ and the major conclusions are summarized in $\$ 5$. 


\section{Carbon Depletion Inferred from the Kramers-Kronig Relation}

Among the 15 Galactic sightlines studied by Parvathi et al. (2012) and the six sightlines studied by Sofia et al. (2011), we select 16 sightlines for which the extinction curves, hydrogen column densities, and gas-phase carbon abundances have been well determined. In Figures 2, 3 we show the UV/optical/near-IR extinction curves of these 16 sources constructed as following, with the extinction parameters taken from Valencic et al. (2004) and Gordon et al. (2009).

The UV extinction at $3.3<\lambda^{-1}<8.7 \mu \mathrm{m}^{-1}$ is derived from the following analytical formula which consists of three parts: a linear background, a Drude profile for the $2175 \AA$ extinction bump, and a far-UV nonlinear rise at $\lambda^{-1}>5.9 \mu \mathrm{m}^{-1}$ (see Valencic et al. 2004, Gordon et al. 2009):

$$
\begin{aligned}
& A_{\lambda} / A_{V}=c_{1}^{\prime}+c_{2}^{\prime} x+c_{3}^{\prime} D\left(x, \gamma, x_{\mathrm{o}}\right)+c_{4}^{\prime} F(x), \\
& D\left(x, \gamma, x_{\mathrm{o}}\right)=\frac{x^{2}}{\left(x^{2}-x_{\mathrm{o}}^{2}\right)^{2}+x^{2} \gamma^{2}} \quad, \\
& F(x)=\left\{\begin{array}{ll}
0, & x<5.9 \mu \mathrm{m}^{-1} \\
0.5392(x-5.9)^{2}+0.05644(x-5.9)^{3}, & x \geq 5.9 \mu \mathrm{m}^{-1}
\end{array},\right.
\end{aligned}
$$

where $x \equiv 1 / \lambda$ is the inverse wavelength in $\mu \mathrm{m}^{-1}, c_{1}^{\prime}$ and $c_{2}^{\prime}$ define the linear background, $c_{3}^{\prime}$ defines the strength of the $2175 \AA$ extinction bump which is approximated by $D\left(x, \gamma, x_{\mathrm{o}}\right)$, a Drude function which peaks at $x_{\mathrm{o}} \approx 4.6 \mu \mathrm{m}^{-1}$ and has a FWHM of $\gamma$, and $c_{4}^{\prime}$ defines the nonlinear far-UV rise. This parametrization was originally introduced by Fitzpatrick \& Massa (1990; hereafter FM90) for the interstellar reddening

$$
E(\lambda-V) / E(B-V)=R_{V}\left(A_{\lambda} / A_{V}-1\right)=c_{1}+c_{2} x+c_{3} D\left(x, \gamma, x_{\mathrm{o}}\right)+c_{4} F(x),
$$

where $E(\lambda-V)=A_{\lambda}-A_{V}, E(B-V)=A_{B}-A_{V}$, and $A_{B}$ is the $B$-band extinction. It is easy to find the relationship between the FM90 parameters and that of Valencic et al. (2004) and Gordon et al. (2009):

$$
c_{j}^{\prime}=\left\{\begin{array}{lr}
c_{j} / R_{V}+1, & j=1 \\
c_{j} / R_{V}, & j=2,3,4,
\end{array}\right.
$$

where $R_{V} \equiv A_{V} / E(B-V)$ is the total-to-selective extinction ratio. The $c_{j}^{\prime}$ parameters of the selected 16 sources are taken from Valencic et al. (2004) and Gordon et al. (2009). Finally, for a given $R_{V}$, the optical/near-IR extinction at $0.3<\lambda^{-1}<3.3 \mu \mathrm{m}^{-1}$ is computed from the CCM parametrization (see Cardelli et al. 1989). We then smoothly join the UV extinction at $\lambda^{-1}>3.3 \mu \mathrm{m}^{-1}$ to the optical/near-IR extinction. 
Let $A_{\text {int }} \equiv \int_{0}^{\infty} A_{\lambda} / N_{\mathrm{H}} d \lambda$ be the extinction integrated over all wavelengths. The total volume of carbon dust per $\mathrm{H}$ nucleon $\left(V_{\mathrm{C}} / \mathrm{H}\right)$ can be derived from the Kramers-Kronig relation of Purcell (1969; see eq. 3) if we assume that the extinction is caused by silicate dust and carbon dust

$$
\frac{V_{\mathrm{C}}}{\mathrm{H}}=\left(\frac{1}{F_{\mathrm{C}}}\right)\left(\frac{A_{\mathrm{int}}}{1.086 \times 3 \pi^{2}}-F_{\mathrm{sil}} \frac{V_{\mathrm{sil}}}{\mathrm{H}}\right),
$$

where $F_{\mathrm{C}}$ and $F_{\text {sil }}$ are respectively the dimensionless factor $F$ seen in eq. 3 for carbon dust and silicate dust. For moderately elongated grains, $F_{\text {sil }} \approx 0.7$ for silicate materials and $F_{\mathrm{C}} \approx 1.25$ for conducting, graphitic materials (see Figure 1). For the selected 16 sources, the extinction per $\mathrm{H}$ nucleon $A_{\lambda} / N_{\mathrm{H}}$ is only known over a limited range of wavelengths. Therefore, we first use eq.9 to extrapolate the UV extinction of Valencic et al. (2004) to $\lambda=912 \AA 99$ For $0.001<\lambda^{-1}<0.3 \mu \mathrm{m}^{-1}$, we adopt the model $A_{\lambda} / N_{\mathrm{H}}$ values of WD01 and Li \& Draine (2001b) for the diffuse ISM. This is justified since the near- and midIR extinction at $\lambda>0.9 \mu \mathrm{m}$ does not seem to vary much among different environments (see Draine 2003a, Wang \& Jiang 2014, Wang et al. 2013, 2014). In this way, we obtain $A_{\mathrm{int}}^{\prime}=\int_{912 \mathrm{~A}}^{1000 \mu \mathrm{m}} A_{\lambda} / N_{\mathrm{H}} d \lambda$ for each source (see Table 1). Since $A_{\mathrm{int}}^{\prime}<A_{\mathrm{int}}$, the volume of carbon dust per $\mathrm{H}$ nucleon $\left(V_{\mathrm{C}} / \mathrm{H}\right)$ derived from eq.14 is a lower limit if we replace $A_{\text {int }}$ by $A_{\text {int }}^{\prime}$. In the following, unless otherwise stated, by $A_{\text {int }}$ we really mean $A_{\text {int }}^{\prime}$.

What is the silicate dust volume per $\mathrm{H}$ nucleon $V_{\text {sil }} / \mathrm{H}$ ? As shown in $\$ 1$, this depends on the assumed interstellar $\mathrm{Fe}, \mathrm{Mg}$ and $\mathrm{Si}$ abundances. In the literature, the proto-Sun abundances (Lodders 2003), the solar abundances (Asplund et al. 2009), the abundances of young F and G stars (Sofia \& Meyer 2001) and/or unevolved early B-type stars (Przybilla et al. 2008) have been adopted to represent the interstellar standard abundances. In this work we will only consider the proto-Sun abundances and the abundances of early B stars since the solar abundances and the abundances of young $\mathrm{F}$ and $\mathrm{G}$ stars are intermediate between that of the proto-Sun and B stars (see Footnote-1). The proto-Sun abundances of $\mathrm{Fe}, \mathrm{Mg}$ and Si result in $V_{\text {sil }} / \mathrm{H} \approx 3.17 \times 10^{-27} \mathrm{~cm}^{3} \mathrm{H}^{-1}$ while the B-star abundances lead to $V_{\text {sil }} / \mathrm{H} \approx 2.52 \times 10^{-27} \mathrm{~cm}^{3} \mathrm{H}^{-1}$. Assuming the carbon dust to be graphite 10 we determine $V_{\mathrm{C}} / \mathrm{H}$ from eq.14 for each sightline. The required carbon depletion is

$$
[\mathrm{C} / \mathrm{H}]_{\text {dust }} \approx\left(V_{\mathrm{C}} / \mathrm{H}\right) \rho_{\mathrm{C}} / 12 m_{\mathrm{H}}
$$

\footnotetext{
${ }^{9}$ Gordon et al. (2009) studied the extinction curves of 75 Galactic sightlines obtained with FUSE at $905<\lambda<1187 \AA$ and the International Ultraviolet Explorer (IUE) at $1150<\lambda<3300 \AA$. They found that the extrapolation of the UV extinction at $3.3<\lambda^{-1}<8.7 \mu \mathrm{m}^{-1}$ obtained by IUE is generally consistent with the far-UV extinction at $8.4<\lambda^{-1}<11 \mu \mathrm{m}^{-1}$ obtained by FUSE.

${ }^{10}$ As shown in Figure 1, for moderately elongated or flattened spheroids the $F_{\mathrm{C}}$ factors are essentially the same for graphite and amorphous carbon. Therefore, the results derived from eq.14 also hold for amorphous carbon.
} 
where $\rho_{\mathrm{C}}$ is the mass density of the carbon dust $\left(\rho_{\mathrm{C}} \approx 2.24 \mathrm{~g} \mathrm{~cm}^{-3}\right.$ for graphite).

In Table 1 we list the total carbon volume $V_{\mathrm{C}} / \mathrm{H}$ and carbon depletion $[\mathrm{C} / \mathrm{H}]_{\text {dust }}$ per $\mathrm{H}$ atom required to account for the observed extinction for each sightline. For a given set of interstellar $\mathrm{Fe}, \mathrm{Mg}$ and Si reference abundances, the uncertainties on $[\mathrm{C} / \mathrm{H}]_{\text {dust }}$ mainly arise from $F_{\mathrm{C}}, F_{\text {sil }}$ and $[\mathrm{Si} / \mathrm{H}]_{\mathrm{ISM}}$. For moderately elongated or flattened spheroids, the $F_{\mathrm{C}}$ and $F_{\text {sil }}$ values adopted here can vary at most $\sim 20 \%$. For a given reference standard (proto-Sun or B stars), the uncertainty on $[\mathrm{Si} / \mathrm{H}]_{\mathrm{ISM}}$ is within $\sim 5 \%$. Therefore, the uncertainties on $[\mathrm{C} / \mathrm{H}]_{\text {dust }}$ are within $\sim 25 \%$. We note that, with $A_{V} \approx 2.16 \pm 0.04$ mag (Gordon et al. 2009) and $N_{\mathrm{H}} \approx 1.78 \times 10^{21} \mathrm{~cm}^{-2} \mathrm{H}$ (Cartledge et al. 2004), the line of sight toward HD 206773 is very dusty in spite it has a large gas-phase carbon abundance $\left([\mathrm{C} / \mathrm{H}]_{\text {gas }} \approx 464 \pm 57 \mathrm{ppm}\right.$; Parvathi et al. 2012). With $A_{V} / N_{\mathrm{H}} \approx 1.12 \times 10^{-21} \mathrm{mag} \mathrm{cm}^{2} \mathrm{H}^{-1}$, the HD 206773 sightline is twice as dusty as the Galactic diffuse ISM of which $A_{V} / N_{\mathrm{H}} \approx 5.3 \times 10^{-22} \mathrm{mag} \mathrm{cm}^{2} \mathrm{H}^{-1}$. Therefore, one requires an unusually large carbon depletion $\left([\mathrm{C} / \mathrm{H}]_{\text {dust }} \approx 733 \mathrm{ppm}\right.$ if the proto-Sun Fe, Mg, and Si abundances are adopted, or $[\mathrm{C} / \mathrm{H}]_{\text {dust }} \approx 774 \mathrm{ppm}$ if the B-star Fe, $\mathrm{Mg}$, and Si abundances are adopted), much larger than that of the other sightlines considered in this work. In the following, unless otherwise stated, we will not include HD 206773 for correlation studies.

In Figure 4 we plot the carbon depletion $[\mathrm{C} / \mathrm{H}]_{\text {dust }}$ as a function of $c_{3}^{\prime}$, the strength of the $2175 \AA$ extinction bump. It can be seen from Figure 4 that $[\mathrm{C} / \mathrm{H}]_{\text {dust }}$ shows an increasing trend with $c_{3}^{\prime}$, with a Pearson linear correlation coefficient of $R \approx 0.60$, and a Kendall correlation coefficient of $\tau \approx 0.39$ and a corresponding probability $p \approx 0.04$ of a chance correlation 11 This is independent of the assumed interstellar Fe, Mg and Si reference abundances which would only affect the intercept. In Figure 5 we plot the carbon depletion $[\mathrm{C} / \mathrm{H}]_{\text {dust }}$ as a function of $c_{4}^{\prime}$, the strength of the nonlinear far-UV extinction rise. Figure 5 shows no detectable correlation between $[\mathrm{C} / \mathrm{H}]_{\text {dust }}$ and $c_{4}^{\prime}$ for which the Pearson correlation coefficient is $R \approx-0.20$ and the Kendall $\tau$ is $\approx-0.01$ and $p \approx 0.96$. Again, these correlation coefficients are independent of the assumed interstellar Fe, Mg and Si reference abundances.

\section{Carbon Depletion Inferred from the Extinction Modeling}

The carbon depletion $[\mathrm{C} / \mathrm{H}]_{\text {dust }}$ derived from the Kramers-Kronig relation is independent of any dust models except we just need to assume that the observed extinction is caused by

\footnotetext{
${ }^{11}$ We set the level of confidence to be $95 \%$ (i.e., Kendall's $\alpha=0.05$ ).
} 
silicate dust and carbon dust 12

We now would like to derive the carbon depletion from fitting the observed extinction curve for each sightline. We consider the silicate-graphite interstellar grain model which consists of two separate dust components: amorphous silicate and graphite (Mathis et al. 1977, Draine \& Lee 1984). We adopt an exponentially-cutoff power-law size distribution for both components: $d n_{i} / d a=n_{\mathrm{H}} B_{i} a^{-\alpha_{i}} \exp \left(-a / a_{c, i}\right)$ for the size range of $50 \AA<a<2.5 \mu \mathrm{m}$, where $a$ is the spherical radius of the dust, $n_{\mathrm{H}}$ is the number density of $\mathrm{H}$ nuclei, $d n_{i}$ is the number density of dust of type $i$ with radii in the interval $[a, a+d a], \alpha_{i}$ and $a_{c, i}$ are respectively the power index and exponential cutoff size for dust of type $i$, and $B_{i}$ is the constant related to the total amount of dust of type $i$. The total extinction per $\mathrm{H}$ column at wavelength $\lambda$ is given by

$$
A_{\lambda} / N_{\mathrm{H}}=1.086 \sum_{i} \int d a \frac{1}{n_{\mathrm{H}}} \frac{d n_{i}}{d a} C_{\mathrm{ext}, i}(a, \lambda),
$$

where the summation is over the two grain types (i.e., silicate and graphite), $N_{\mathrm{H}} \equiv \int n_{\mathrm{H}} d l$ is the $\mathrm{H}$ column density which is the $\mathrm{H}$ number density integrated over the line of sight $l$, and $C_{\text {ext, } i}(a, \lambda)$ is the extinction cross section of grain type $i$ of size $a$ at wavelength $\lambda$ which can be calculated from Mie theory (Bohren \& Huffman 1983) using the dielectric functions of "astronomical" silicate and graphite of Draine \& Lee (1984).

In fitting the extinction curve, for a given sightline, we have six parameters: the size distribution power indices $\alpha_{\mathrm{S}}$ and $\alpha_{\mathrm{C}}$ for silicate and graphite, respectively; the exponential cutoff sizes $a_{c, \mathrm{~S}}$ and $a_{c, \mathrm{C}}$, respectively; and $B_{\mathrm{S}}$ and $B_{\mathrm{C}}$. We derive the silicon and carbon depletions from

$$
\begin{aligned}
& {[\mathrm{Si} / \mathrm{H}]_{\text {dust }}=\left(n_{\mathrm{H}} B_{\mathrm{S}} / 172 m_{\mathrm{H}}\right) \int d a(4 \pi / 3) a^{3} \rho_{\mathrm{sil}} a^{-\alpha_{\mathrm{S}}} \exp \left(-a / a_{c, \mathrm{~S}}\right)} \\
& {[\mathrm{C} / \mathrm{H}]_{\text {dust }}=\left(n_{\mathrm{H}} B_{\mathrm{C}} / 12 m_{\mathrm{H}}\right) \int d a(4 \pi / 3) a^{3} \rho_{\mathrm{gra}} a^{-\alpha_{\mathrm{C}}} \exp \left(-a / a_{c, \mathrm{C}}\right)}
\end{aligned}
$$

where we assume a stoichiometric composition of $\mathrm{MgFeSiO}_{4}$ for amorphous silicate.

For a given sightline, we seek the best fit to the extinction between $0.3 \mu \mathrm{m}^{-1}$ and $8 \mu \mathrm{m}^{-1}$ by varying the size distribution power indices $\alpha_{\mathrm{S}}$ and $\alpha_{\mathrm{C}}$, and the upper cutoff size parameters $a_{c, \mathrm{~S}}$ and $a_{c, \mathrm{C}}$. Following WD01, we evaluate the extinction at 100 wavelengths $\lambda_{i}$, equally

\footnotetext{
${ }^{12}$ The correlation coefficients derived in $\oint_{2}$ and shown in Figure 4 remain the same for both graphite and amorphous carbon. The required carbon depletion $[\mathrm{C} / \mathrm{H}]_{\text {dust }}$ would decrease by a factor of $\rho_{\text {gra }} / \rho_{\text {ac }}$, where $\rho_{\mathrm{ac}} \approx 1.8 \mathrm{~g} \mathrm{~cm}^{-3}$ is the mass density of amorphous carbon.
} 
spaced in $\ln \lambda$. We use the Levenberg-Marquardt method (Press et al. 1992) to minimize $\chi^{2}$ which gives the error in the extinction fit:

$$
\chi^{2}=\sum_{i} \frac{\left(\ln A_{\mathrm{obs}}-\ln A_{\mathrm{mod}}\right)^{2}}{\sigma_{i}^{2}},
$$

where $A_{\text {obs }}\left(\lambda_{i}\right)$ is the observed extinction at wavelength $\lambda_{i}, A_{\text {mod }}\left(\lambda_{i}\right)$ is the extinction computed for the model at wavelength $\lambda_{i}$ (see eq.16), and the $\sigma_{i}$ are weights. Following WD01, we take the weights $\sigma_{i}^{-1}=1$ for $1.1<\lambda^{-1}<8 \mu \mathrm{m}^{-1}$ and $\sigma_{i}^{-1}=1 / 3$ for $\lambda^{-1}<1.1 \mu \mathrm{m}^{-1}$.

In Figures 2, 3 we show the model fits. It can be seen from these figures that a simple mixture of silicate and graphite closely reproduces the observed UV/optical/near-IR extinction of all 16 sightlines. The model parameters are tabulated in Table 2. In Figure 6 we examine the correlations between the strength of the $2175 \AA$ extinction bump $\left(c_{3}^{\prime}\right)$ with the carbon $\left([\mathrm{C} / \mathrm{H}]_{\text {dust }}\right)$ and silicon $\left([\mathrm{Si} / \mathrm{H}]_{\text {dust }}\right)$ depletions derived from fitting the observed extinction (see eqs.17, 18). With a Pearson correlation coefficient of $R \approx 0.83$ and a Kendall $\tau \approx 0.49$ and $p \approx 0.01$, we see that the carbon depletion correlates with the $2175 \AA$ bump, while the silicon depletion does not correlate with the $2175 \AA$ bump. We have also explored the relations between the strength of the nonlinear far-UV extinction rise $\left(c_{4}^{\prime}\right)$ and the carbon and silicon depletions. As shown in Figure 7, we do not see any correlation.

\section{Discussion}

Interstellar dust plays an important role in various physical and chemical processes that take place in the ISM. Dust extinction remains the most direct way to investigate the properties of interstellar dust since Trumpler (1930) first established the existence of solid particles in interstellar space through the discovery of color excess. However, practically most of the extinction modeling efforts have been so far directed to the Galactic average extinction curve which is obtained by averaging over many clouds of different gas and dust properties. An investigation of dust extinction toward individual clouds is of special importance.

In this work we explore the extinction and dust depletion of 16 individual sightlines of which $R_{V}$ ranges from $\sim 2.4$ to $\sim 4.3$ and the average hydrogen density $\left\langle n_{\mathrm{H}}\right\rangle$ ranges over nearly three orders of magnitudes (see Figure 8). The hydrogen column density and the gasphase carbon abundance are known for these sightlines, with the latter determined from the strong $1334 \AA$ CII absorption line (Parvathi et al. 2012, Sofia et al. 2011). Unlike Cardelli et al. (1996) who derived the interstellar gas-phase carbon abundance to be $[\mathrm{C} / \mathrm{H}]_{\text {gas }} \approx$ $(140 \pm 20) \mathrm{ppm}$ and found that $[\mathrm{C} / \mathrm{H}]_{\text {gas }}$ shows no dependence on the physical condition of the gas, for the sightlines studied by Parvathi et al. (2012) and Sofia et al. (2011), the gas- 
phase $[\mathrm{C} / \mathrm{H}]_{\text {gas }}$ appears to show a decreasing trend for increasing values of $\left\langle n_{\mathrm{H}}\right\rangle$ (see Figure 8 , also see Figure 3 in Parvathi et al. 2012) 13

The lack of any statistically significant variation in the observed gas-phase $[\mathrm{C} / \mathrm{H}]_{\text {gas }}$ among the six sightlines studied by Cardelli et al. (1996) is in strong contrast to the appreciable variation of $[\mathrm{C} / \mathrm{H}]_{\text {gas }}$ with $\left\langle n_{\mathrm{H}}\right\rangle$ seen in the sightlines studied in this work. As shown in Figure 8, the solid-phase silicon $\left([\mathrm{Si} / \mathrm{H}]_{\text {dust }}\right)$ and carbon $\left([\mathrm{C} / \mathrm{H}]_{\text {dust }}\right)$ abundances derived from fitting the observed extinction also exhibit substantial variations with $\left\langle n_{\mathrm{H}}\right\rangle$. The solid-phase carbon abundance $\left([\mathrm{C} / \mathrm{H}]_{\text {dust }}\right)$ determined from the wavelength-integrated extinction $\left(A_{\text {int }}\right)$ based on the Kramers-Kronig relation varies by a factor of $>10$ among these 16 sightlines. Therefore, the total abundance of carbon, the sum of the components residing in the gas and in the dust, varies significantly among these sightlines. This raises a question: is there indeed a reference standard for the interstellar carbon abundance?

As shown in Table 1, the analysis of the wavelength-integrated extinction based on the Kramers-Kronig relation shows that five of the 16 sources require in total a carbon abundance (i.e., $[\mathrm{C} / \mathrm{H}]_{\text {dust }}+[\mathrm{C} / \mathrm{H}]_{\text {gas }}$ ) exceeding $\sim 500 \mathrm{ppm}$, much higher than that of the young $\mathrm{F}$ and $\mathrm{G}$ stars $\left([\mathrm{C} / \mathrm{H}]_{\star} \approx 355 \pm 82 \mathrm{ppm}\right.$, Sofia \& Meyer 2001), the proto-Sun $\left([\mathrm{C} / \mathrm{H}]_{\odot} \approx 288 \pm 27 \mathrm{ppm}\right.$, Lodders 2003), the Sun $\left([\mathrm{C} / \mathrm{H}]_{\odot} \approx 269 \pm 31 \mathrm{ppm}\right.$, Asplund et al. 2009), and the early B stars $\left([\mathrm{C} / \mathrm{H}]_{\star} \approx 214 \pm 20 \mathrm{ppm}\right.$, Przybilla et al. 2008), which are often taken to be the interstellar carbon reference abundance. This is also true for the carbon depletion derived from fitting the observed extinction with the silicate-graphite model. Mathis (1996) argued that a dust model may require fewer $\mathrm{C}, \mathrm{Si}, \mathrm{Mg}$ and Fe atoms to account for the observed extinction if the dust has a fluffy, porous structure since fluffy grains are more effective in absorbing and scattering the UV/optical starlight than compact grains (on a per unit mass basis). However, as demonstrated in Li (2005), fluffy dust is not able to appreciably reduce the consumption of dust-forming atoms. As can be seen in eq. 3, although the dust volume $V_{\text {dust }}$ does increase when the dust has voids and becomes porous, the $F$-factor decreases and therefore, for a given amount of dust material, the wavelength-integrated extinction $\int A_{\lambda} / N_{\mathrm{H}} d \lambda$ does not change much (see Figures 1,2 in Li 2005). Alternatively, one may argue that the gas-phase $[\mathrm{C} / \mathrm{H}]_{\text {gas }}$ abundances derived from the strong CII absorption line at $\lambda \approx 1334 \AA$ may be overestimated since accurate measures of the absorption in the wings of this line is very difficult. However, the weak CII line at $\lambda \approx 2325 \AA$ gives even higher $[\mathrm{C} / \mathrm{H}]_{\text {gas }}$ abundances (Sofia et al. 2004, Parvathi et al. 2010). Therefore, it seems real that the total $\mathrm{C} / \mathrm{H}$ abundance of some of our 16 sigtlines is well above that of the popular interstellar reference standards.

\footnotetext{
${ }^{13}$ Cardelli et al. (1996) determined $[\mathrm{C} / \mathrm{H}]_{\text {gas }}$ from the weak intersystem line of $\left.\mathrm{CII}\right]$ at $2325 \AA$ obtained with the HST Goddard High Resolution Spectrograph (GHRS) for six sightlines toward stars within $600 \mathrm{pc}$ of the Sun. These sightlines include examples exhibiting a wide range of extinction variation.
} 
In fitting the observed extinction (see $\$ 3$ and Table 2), seven out of the 16 sources require $[\mathrm{Si} / \mathrm{H}]_{\text {dust }}$ to exceed the proto-Sun silicon abundance by $>50 \%$, three of them are within $\sim 20 \%$ of the proto-Sun silicon abundance, and six of them require less silicon than that of the proto-Sun. The variations in the silicon and carbon depletions toward different sightlines imply various physical processing (e.g., growth, coagulation, destruction, and photo-processing) of interstellar dust and variations in the composition within the Milky Way (Parvathi et al. 2012).

Parvathi et al. (2012) found no correlation between the carbon depletion $[\mathrm{C} / \mathrm{H}]_{\text {dust }}$ and the strength of the $2175 \AA$ extinction bump $\left(c_{3}^{\prime}\right)$, while in this work we find $[\mathrm{C} / \mathrm{H}]_{\text {dust }}$ correlates with $c_{3}^{\prime}$ (see $\$ 2$ and $\$ 3$ ). The major difference between our approaches and Parvathi et al. (2012) is that they assumed a reference standard of $[\mathrm{C} / \mathrm{H}]_{\mathrm{ISM}}=464 \mathrm{ppm}$ for all sightlines, while the approaches taken by us which make use of the Kramers-Kronig relation (see \$2) or model the observed extinction (see \$3) result in considerable variation in the total $\mathrm{C} / \mathrm{H}$ abundance. We note that for the Kramers-Kronig-relation-based approach we have assumed a reference standard for $\mathrm{Si}, \mathrm{Mg}$ and $\mathrm{Fe}$ (see 92 ). One may argue that the correlation between $[\mathrm{C} / \mathrm{H}]_{\text {dust }}$ and $c_{3}^{\prime}$ derived from this approach may not be real: with $[\mathrm{Si} / \mathrm{H}]_{\text {dust }},[\mathrm{Mg} / \mathrm{H}]_{\text {dust }}$ and $[\mathrm{Fe} / \mathrm{H}]_{\text {dust }}$ fixed at a reference standard, one might expect the required $\mathrm{C} / \mathrm{H}$ depletion to increase with the extinction. However, this is not true. With $[\mathrm{Si} / \mathrm{H}]_{\text {dust }},[\mathrm{Mg} / \mathrm{H}]_{\text {dust }}$ and $[\mathrm{Fe} / \mathrm{H}]_{\text {dust }}$ fixed, we do expect $[\mathrm{C} / \mathrm{H}]_{\text {dust }}$ to increase with $A_{\lambda} / N_{\mathrm{H}}$, but we do not necessarily expect $[\mathrm{C} / \mathrm{H}]_{\text {dust }}$ to increase with $c_{3}^{\prime}$ since the latter is a quantity normalized by $A_{V}$ and therefore the common correlation of $[\mathrm{C} / \mathrm{H}]_{\text {dust }}$ and $c_{3}^{\prime}$ with $A_{V}$ is cancelled. This can be tested by fixing $[\mathrm{C} / \mathrm{H}]_{\mathrm{ISM}}$ and determining the $\mathrm{Si} / \mathrm{H}$ depletion from the Kramers-Kronig relation and then examining the correlation between $c_{3}^{\prime}$ and $[\mathrm{Si} / \mathrm{H}]_{\text {dust }}$. Assuming the same total $\mathrm{C} / \mathrm{H}$ abundance of $[\mathrm{C} / \mathrm{H}]_{\mathrm{ISM}}$ for all 16 sightlines, the carbon dust volume per $\mathrm{H}$ nucleon for a given sightline can be derived from its gas-phase $\mathrm{C} / \mathrm{H}$ abundance:

$$
\frac{V_{\mathrm{C}}}{\mathrm{H}}=\left\{[\mathrm{C} / \mathrm{H}]_{\mathrm{ISM}}-[\mathrm{C} / \mathrm{H}]_{\text {gas }}\right\} \times 12 m_{\mathrm{H}} / \rho_{\mathrm{C}} .
$$

We use the Kramers-Kronig relation to determine the silicate volume per $\mathrm{H}$ nucleon from the wavelength-integrated extinction:

$$
\frac{V_{\mathrm{sil}}}{\mathrm{H}}=\left(\frac{1}{F_{\mathrm{sil}}}\right)\left(\frac{A_{\text {int }}}{1.086 \times 3 \pi^{2}}-F_{\mathrm{C}} \frac{V_{\mathrm{C}}}{\mathrm{H}}\right) .
$$

Then we derive the required $\mathrm{Si} / \mathrm{H}$ depletion from

$$
[\mathrm{Si} / \mathrm{H}]_{\text {dust }}=\left(V_{\text {sil }} / \mathrm{H}\right) \rho_{\text {sil }} / \mu_{\text {sil }} m_{\mathrm{H}},
$$

where $\mu_{\text {sil }}=172$ is the molecular weight for $\mathrm{MgFeSiO}_{4}$. Following Parvathi et al. (2012), we adopt $[\mathrm{C} / \mathrm{H}]_{\mathrm{ISM}}=464 \mathrm{ppm}$. In Figure 9 we plot $[\mathrm{Si} / \mathrm{H}]_{\text {dust }}$ against $c_{3}^{\prime}$ and $c_{4}^{\prime}$ and see no 
correlation. This justifies the correlation between $[\mathrm{C} / \mathrm{H}]_{\text {dust }}$ and $c_{3}^{\prime}$ derived in the KramersKronig-relation-based approach presented in $\$ 2$ which assumes fixed $[\mathrm{Si} / \mathrm{H}]_{\text {dust }},[\mathrm{Mg} / \mathrm{H}]_{\text {dust }}$ and $[\mathrm{Fe} / \mathrm{H}]_{\text {dust }}$ depletions.

We note that although the carbon abundance $[\mathrm{C} / \mathrm{H}]_{\mathrm{ISM}}$ is underabundant in accounting for the observed extinction, no matter what reference standard (proto-Sun, Sun, early B stars, or young $\mathrm{F}$ and $\mathrm{G}$ stars) is adopted, the oxygen abundance remains largely unaccounted for (see Whittet 2010). Recently, Poteet et al. (2015) argued that the missing oxygen may reside in large, micrometer-sized $\mathrm{H}_{2} \mathrm{O}$ ice grains which are nearly opaque to IR radiation. Possible evidence for the presence of $\mu \mathrm{m}$-sized interstellar grains have been found by Wang et al. (2014), who modeled the mid-IR extinction toward a variety of interstellar environments using a population of $\mu \mathrm{m}$-sized grains. Furthermore, the recent analysis of three possibly interstellar grains returned by the Stardust spacecraft also provides tentative evidence for the presence of $\mu \mathrm{m}$-sized grains in the ISM (Sterken et al. 2014, Westphal et al. 2014). These $\mu \mathrm{m}$-sized grains, although gray in the UV/optical/near-IR, will contribute to the wavelengthintegrated extinction $A_{\text {int }}$ and therefore reduce the consumption of silicon and carbon (i.e., decreasing $[\mathrm{C} / \mathrm{H}]_{\text {dust }}$ and $[\mathrm{Si} / \mathrm{H}]_{\text {dust }}$ ).

The correlation between the $2175 \AA$ bump strength and the carbon depletion $[\mathrm{C} / \mathrm{H}]_{\text {dust }}$ inferred both from the Kramers-Kronig relation and from fitting the observed extinction with a specific dust model supports the hypothesis of carbonaceous grains (e.g., graphite or PAHs) as the possible carriers of the $2175 \AA$ bump.14 The lack of correlation between the silicon depletion and the $2175 \AA$ bump argues against small silicates or $(\mathrm{Mg}, \mathrm{Si})$ oxides as its carrier (see Duley 1985).

Finally, we examine the relations between the carbon and silicon depletions with $R_{V}^{-1}$. As shown in Figure10, the carbon depletion appears to moderately correlate with $R_{V}^{-1} \cdot R_{V}$ is roughly indicative of the dust size: the smaller $R_{V}$ is, the steeper is the far-UV extinction and the richer in small dust is the dust size distribution. This suggests that the far-UV extinction is more likely produced by small carbon dust than by small silicate dust, probably arising from the blue wing of the $\sigma-\sigma^{*}$ electronic absorption profile of graphitic materials. We note that $R_{V}$ provides a better characterization of the far-UV extinction than $c_{4}^{\prime}$ as the latter only accounts for the far-UV nonlinear extinction rise while the linear background $c_{1}^{\prime}+c_{2}^{\prime} x$ surely also contributes to the far-UV extinction. In Figure 11 we plot the $\mathrm{C} / \mathrm{H}$ and $\mathrm{Si} / \mathrm{H}$ depletions against $c_{2}^{\prime}$. As expected, we do not see any correlation because, similar to $c_{4}^{\prime}, c_{2}^{\prime}$

\footnotetext{
${ }^{14}$ Amorphous carbon (Bussoletti et al. 1987), graphitized hydrogenated amorphous carbon (HAC; Mennella et al. 1996), and nano-sized HAC (Schnaiter et al. 1998) have also been proposed as candidate materials. However, they are not always successful in reproducing the observed invariant peak position and variable strength and width of the $2175 \AA$ bump.
} 
alone does not characterize the far-UV extinction.

\section{Conclusion}

We have studied the extinction and dust depletion in 16 Galactic sightlines in order to probe the role of carbon in the UV extinction, with special attention paid to the $2175 \AA$ extinction bump. These sightlines have their UV/optical/near-IR extinction, gas-phase carbon abundances, and hydrogen column densities well determined, therefore allowing us to quantitatively explore the relations between the silicon and carbon depletions and the UV extinction. Our principal results are as follows:

1. We have derived the carbon depletion $[\mathrm{C} / \mathrm{H}]_{\text {dust }}$ from the Kramers-Kronig relation which relates the wavelength-integrated extinction to the total dust volume. We find that $[\mathrm{C} / \mathrm{H}]_{\text {dust }}$ intends to correlate with the strength of the $2175 \AA$ extinction bump. This is less model-dependent since it does not require the knowledge of the detailed optical properties and size distribution of the dust.

2. We have derived the carbon $[\mathrm{C} / \mathrm{H}]_{\text {dust }}$ and silicon $[\mathrm{Si} / \mathrm{H}]_{\text {dust }}$ depletions from fitting the observed UV/optical/near-IR extinction with a mixture of silicate dust and carbonaceous dust. We find that $[\mathrm{C} / \mathrm{H}]_{\text {dust }}$ correlates with the $2175 \AA$ extinction bump and $[\mathrm{Si} / \mathrm{H}]_{\text {dust }}$ does not correlate with the $2175 \AA$ bump. This supports graphite or PAH molecules as the possible carrier of the $2175 \AA$ bump, while argues against the hypothesis of silicate or $(\mathrm{Mg}, \mathrm{Si})$ oxides as its carrier.

3. A moderate correlation between $[\mathrm{C} / \mathrm{H}]_{\text {dust }}$ and $1 / R_{V}$ is derived from the extinction modeling. This suggests that the far-UV extinction is more likely produced by small carbon dust than by small silicate dust.

We thank B.W. Jiang, Q. Li, B. Mills, J.Y. Seok, and Y.X. Xie for helpful discussions. We are particularly grateful to S. Federman, S. Wang, and the anonymous referee for their suggestions which significantly improved the quality of this paper. We are supported in part by NSF AST-1109039, NNX13AE63G, and NSFC 11173019, 11273022 and the University of Missouri Research Board.

\section{REFERENCES}

Allamandola, L.J., Tielens, A.G.G.M., \& Barker, J.R. 1985, ApJ, 290, L25 
Asplund, M., Grevesse, N., Sauval, A.J., \& Scott, P. 2009, ARA\&A, 47, 481

Bohren, C.F., \& Huffman, D.R. 1983, Absorption and Scattering of Light by Small Particles (New York: Wiley)

Bussoletti, E., Colangeli, L., Borghesi, A., \& Orofino, V. 1987, A\&AS, 70, 257

Cardelli, J.A., Clayton, G.C., \& Mathis, J.S. 1989, ApJ, 345, 245 (CCM)

Cartledge, S.I.B., Lauroesch, J.T., Meyer, D.M., \& Sofia, U.J. 2004, ApJ, 613, 1037

Cartledge, S.I.B., Lauroesch, J.T., Meyer, D.M., \& Sofia, U.J. 2006, ApJ, 641, 327

Cecchi-Pestellini, C., Malloci, G., Mulas, G., Joblin, C., \& Williams, D. A. 2008, A\&A, 486, L25

Chiar, J. E., Tielens, A. G. G. M., Adamson, A. J., \& Ricca, A. 2013, ApJ, 770, 78

Davis, A., Leigh, C.R.A., Hampton, R.N., Saunders, G.A., \& Parker, S.C. 1988, J. Mater. Sci. Lett., 7, 415

Draine, B.T., \& Lee, H.M. 1984, ApJ, 258, 89D

Draine, B.T., \& Malhotra, S. 1993, ApJ, 414, 632

Draine, B.T. 2003a, ARA\&A, 41, 241

Draine, B.T. 2003b, in The Cold Universe, Saas-Fee Advanced Course Vol. 32, ed. D. Pfenniger (Berlin: Springer-Verlag), 213

Duley, W.W., Jones, A.P., \& Williams, D.A. 1989, MNRAS, 236, 709

Duley, W.W. 1985, Ap\&SS, 112, 321

Fitzpatrick, E.L., \& Massa, D. 1990, ApJS, 72, 163

Furton, D. G., Laiho, J. W., \& Witt, A. N. 1999, ApJ, 526, 752

Gordon, K.D., Cartledge, S., \& Clayton, G.C. 2009, ApJ, 705, 1320

Greenberg, J.M., \& Li, A. 1996, A\&A, 309, 258

Henning, Th. 2010, ARA\&A, 48, 21

Hildebrand, R.H., \& Dragovan, M. 1995, ApJ, 450, 663 
Jenkins, E.B. 2009, ApJ, 700, 1299

Joblin, C., Léger, A., \& Martin, P. 1992, ApJ, 393, L79

Jones, A.P., Fanciullo, L., Köhler, M., et al. 2013, A\&A, 558, AA62

Kemper, F., Vriend, W.J., \& Tielens, A.G.G.M. 2004, ApJ, 609, 826

Kim, S.H., Martin, P.G., \& Hendry, P.D. 1994, ApJ, 422, 164

Lee, H.M., \& Draine, B.T. 1985, ApJ, 290, 211

Léger, A., \& Puget, J. 1984, A\&A, 137, L5

Li, A., \& Greenberg, J.M. 1997, A\&A, 323, 566

Li, A., \& Draine, B.T. 2001a, ApJ, 550, L213

Li, A., \& Draine, B.T. 2001b, ApJ, 554, 778

Li, A. 2005, ApJ, 622, 965

Li, M.P., Shi, Q.J., \& Li, A. 2008, MNRAS, 391, L49

Lodders, K. 2003, ApJ, 591, 1220

Mathis, J.S., Rumpl, W., \& Nordsieck, K.H. 1977, ApJ, 217, 425

Mathis, J.S. 1994, ApJ, 422, 176

Mathis, J. S. 1996, ApJ, 472, 643

Mennella, V., Colangeli, L., Palumbo, P., et al. 1996, ApJ, 464, L191

Mulas, G., Zonca, A., Casu, S., \& Cecchi-Pestellini, C. 2013, ApJS, 207, 7

Parvathi, V.S., Sofia, U.J., Murthy, J., \& Babu. B.R.S. 2012, ApJ, 760, 36

Parvathi, V. S., Sofia, U. J., Murthy, J., \& Babu, B. R. S. 2010, Bull. Astron. Soc. India, 38,93

Pendleton, Y. J., \& Allamandola, L. J. 2002, ApJS, 138, 75

Poteet, C. A., Whittet, D. C. B., \& Draine, B. T. 2015, ApJ, 801, 110 
Press, W.H., Teukolsky, S.A., Vetterling, W.T., \& Flannery, B.P. 1992, Numerical Recipes in FORTRAN: The Art of Scientific Computing (2nd ed.; Cambridge: Cambridge Univ. Press)

Przybilla, N., Nieva, M.-F., \& Butler, K. 2008, ApJ, 688, L103

Purcell, E.M. 1969, ApJ, 158, 433

Schnaiter, M., Mutschke, H., Dorschner, J., Henning, T., \& Salama, F. 1998, ApJ, 498, 486

Snow, T.P., \& Witt, A.N. 1996, ApJ, 468, L65

Sofia, U.J., \& Meyer, D.M. 2001, ApJ, 558, L147

Sofia, U.J. 2004, in ASP Conf. Ser. 309, Astrophysics of Dust, ed. A.N. Witt, G.C. Clayton, \& B.T. Draine (San Francisco: ASP), 393

Sofia, U. J., Lauroesch, J. T., Meyer, D. M., \& Cartledge, S. I. B. 2004, ApJ, 605, 272

Sofia, U.J., Parvathi, V.S., Babu, B.R.S., \& Murthy, J. 2011, AJ, 141, 22

Stecher, T.P., \& Donn, B. 1965, ApJ, 142, 1681

Steglich, M., Jäger, C., Rouillé, G., et al. 2010, ApJ, 712, L16

Sterken, V. J., Westphal, A. J., Altobelli, N., et al. 2014, Meteorit. Planet. Sci., 49, 1680

Trumpler, R.J. 1930, PASP, 42, 214

Wang, S., \& Jiang, B.W. 2014, ApJ, 788, L12

Wang, S., Li, A., \& Jiang, B.W. 2014, Planet. Space Sci., 100, 32

Wang, S., Gao, J., Jiang, B.W., Li, A., \& Chen, Y. 2013, ApJ, 773, 30

Weingartner, J.C., \& Draine, B.T. 2001, ApJ, 548, 296 (WD01)

Westphal, A.J., et al. 2014, Science, 345, 786

Whittet, D.C.B. 2003, Dust in the Galactic Environment (2nd ed; Bristol: IoP)

Whittet, D. C. B. 2010, ApJ, 710, 1009

Witt, A. N.,\& Vijh, U. P. 2004, in ASP Conf. Ser. 309, Astrophysics of Dust, ed. A.N. Witt, G.C. Clayton, \& B.T. Draine (San Francisco: ASP), 115 
Valencic, L.A., Clayton, G.C., \& Gordon, K.D. 2004, ApJ, 616, 912

Zhang, K., Jiang, B. W., \& Li, A. 2009, MNRAS, 396, 1247

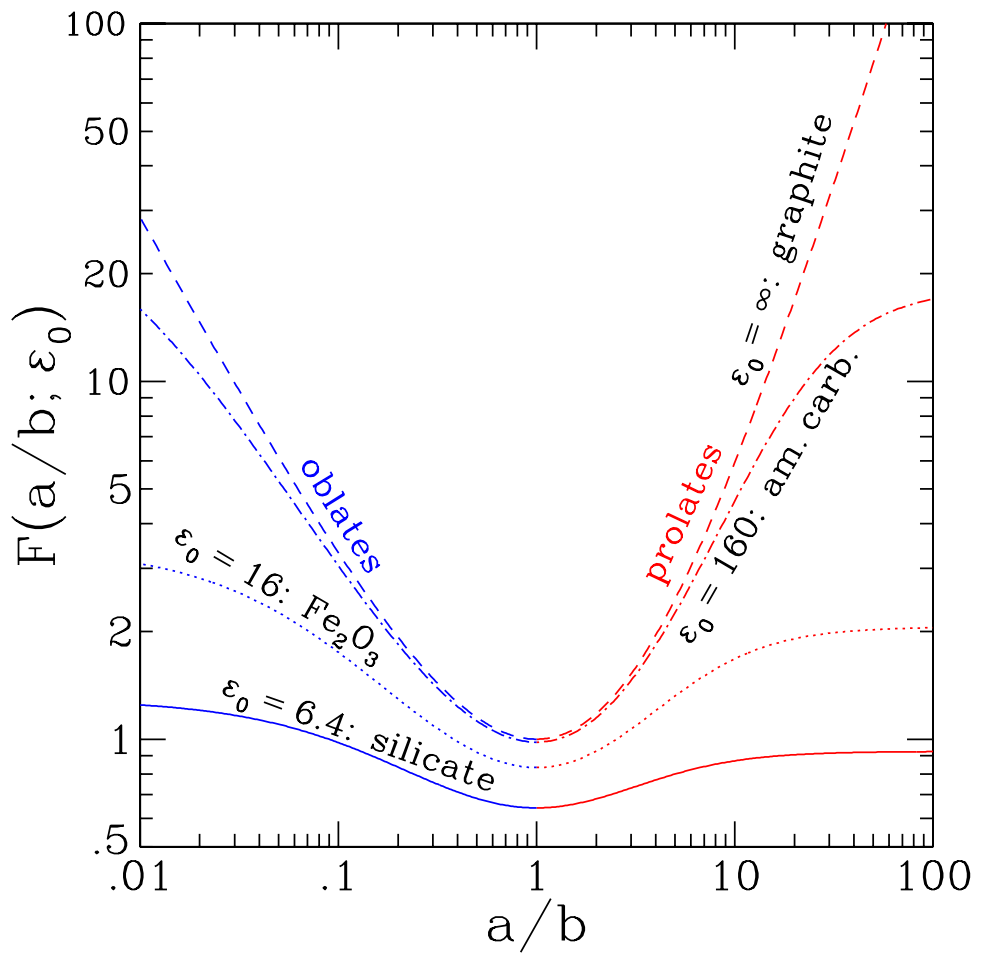

Fig. 1. - The $F\left(\varepsilon_{0}\right.$; shape) factor as a function of the axial ratio $a / b$ for silicate (solid line), $\mathrm{Fe}_{2} \mathrm{O}_{3}$ (dotted line), amorphous carbon (dot-dashed line), and graphite (dashed line) of which the static dielectric constants are approximately $\varepsilon_{0} \approx 6.4,16,160$ and $\infty$ (Li 2005). The grains are taken to be spheroidal with $a$ and $b$ being the semiaxis along and perpendicular to the symmetry axis of the spheroid, respectively. The blue lines are for oblates $(a / b<1)$ and the red lines are for prolates $(a / b>1)$. For modestly elongated $(a / b \lesssim 3)$ or flattened $(b / a \lesssim 3)$ silicate dust, the $F$ factor is always smaller than unity. 

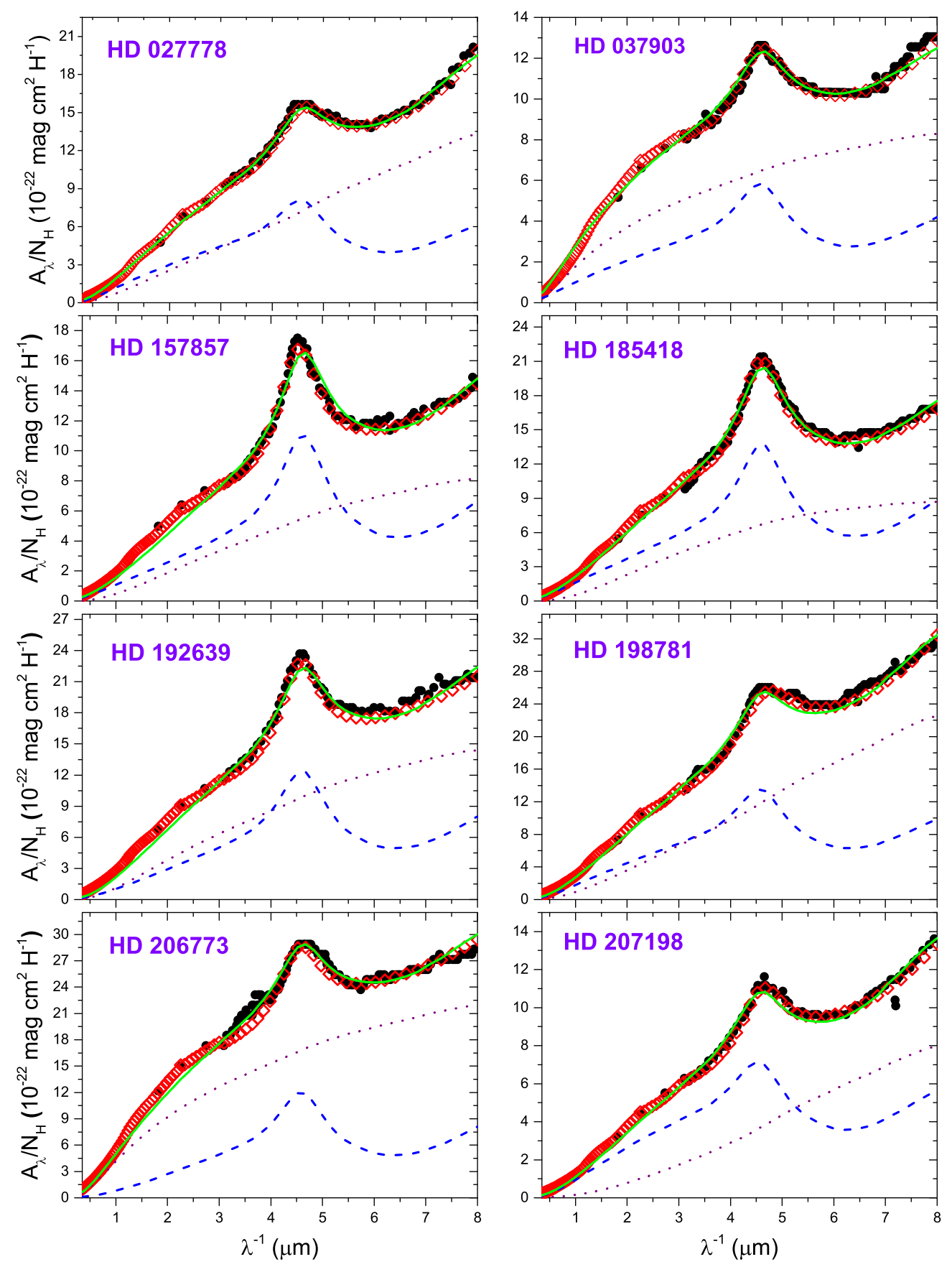

Fig. 2.- Observed and model extinction curves of HD 027778, HD 037903, HD 157857, HD 185418, HD 192639, HD 198781, HD 206773, and HD 207198. The observed extinction curves are represented by the FM90 parametrization at $\lambda^{-1}>3.3 \mu \mathrm{m}^{-1}$ and by the CCM parametrization at $\lambda^{-1}<3.3 \mu \mathrm{m}^{-1}$ (open red diamonds). The filled black circles plot the optical data at $U, B, V$ bands and the IUE data at $3.3<\lambda^{-1}<8.7 \mu \mathrm{m}^{-1}$ (taken from Gordon et al. 2009). The solid green line plots the model extinction curve which is a combination of silicate (dotted purple line) and graphite (dashed blue line). 

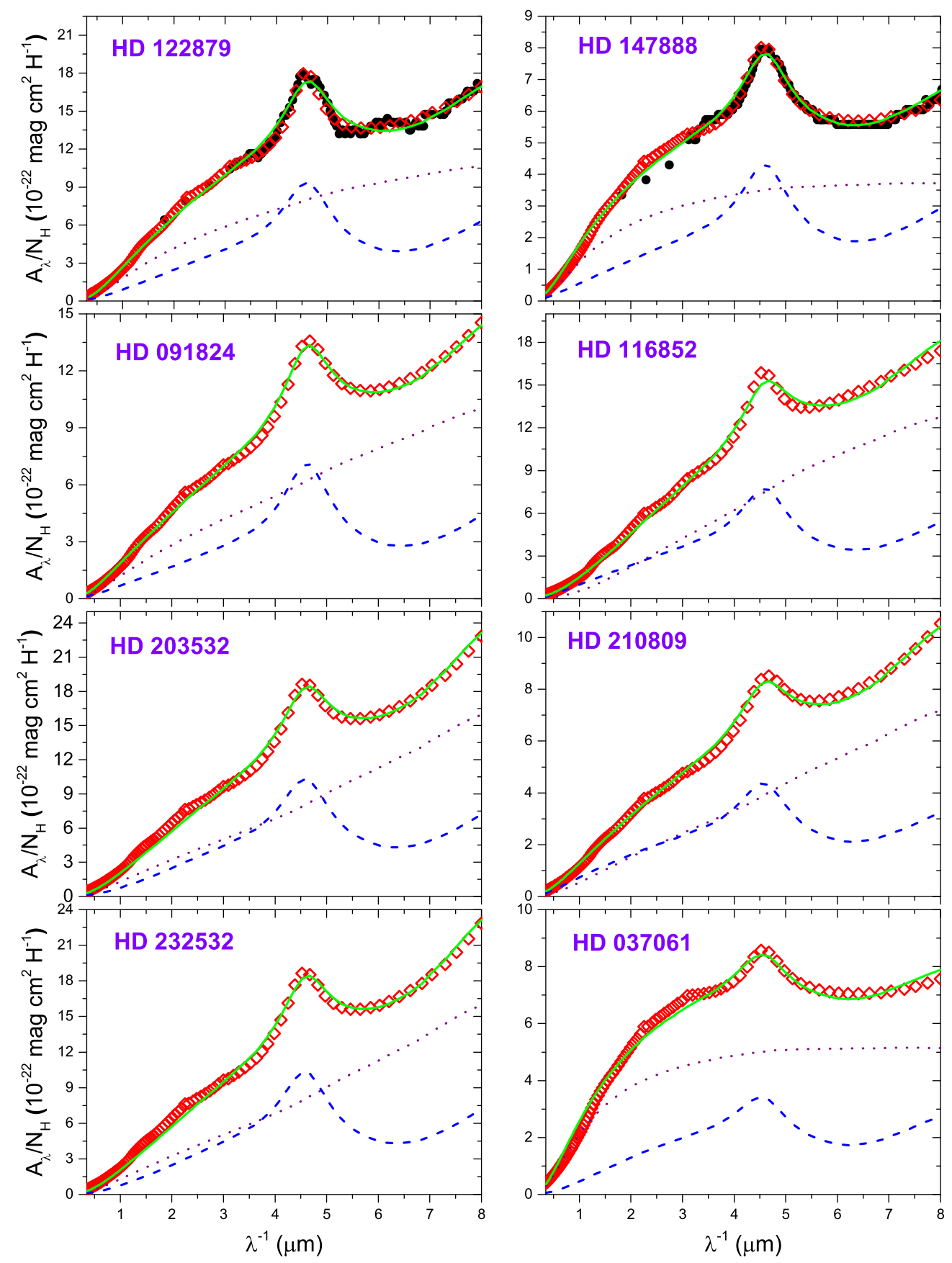

Fig. 3.- Same as Figure 3 but for HD 122879, HD 147888, HD 091824, HD 116852, HD 203532, HD 210809, HD 232532, and HD 037061. For the latter six sources, the optical and IUE data are not shown. 

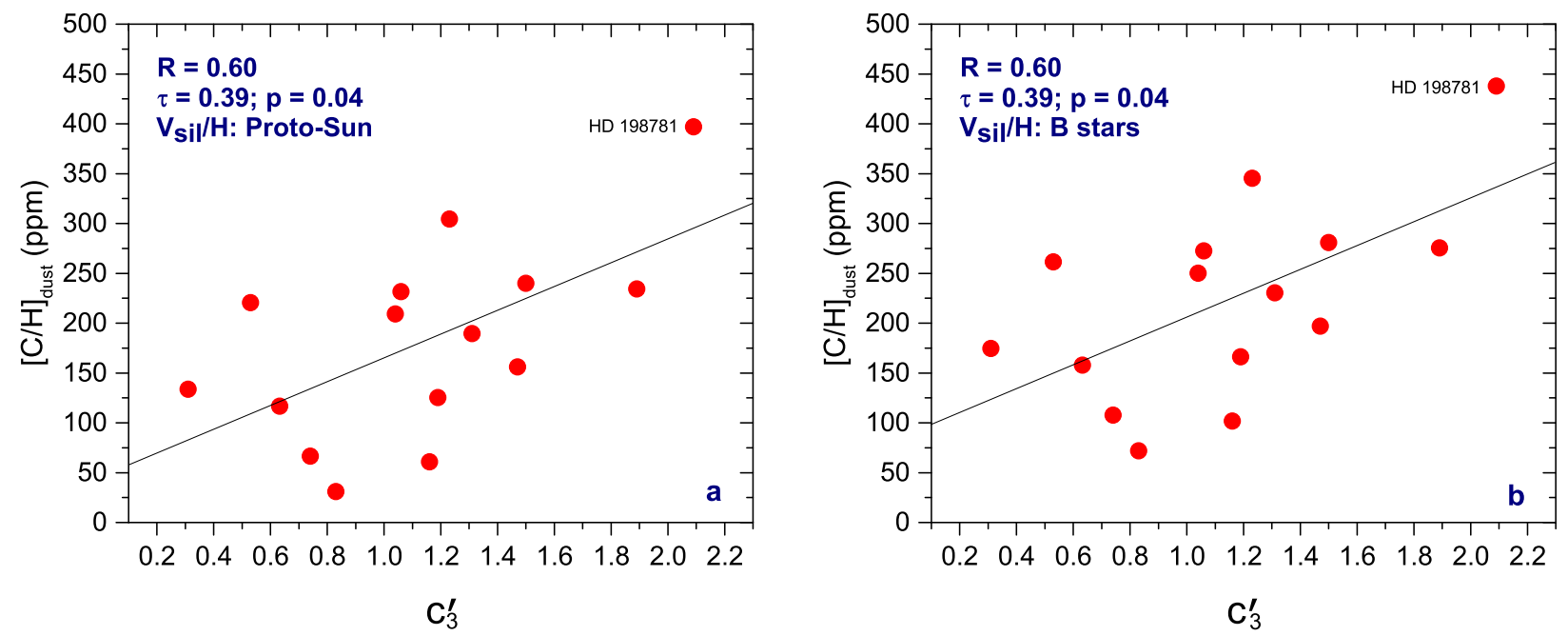

Fig. 4. - Correlation diagrams between the carbon depletion $[\mathrm{C} / \mathrm{H}]_{\text {dust }}$ with the strength of the $2175 \AA$ extinction bump $\left(c_{3}^{\prime}\right)$. The carbon depletion is derived from the Kramers-Kronig relation (see eqs.3114), with the interstellar Fe, Mg and Si abundances taken to be that of proto-Sun (a) or B stars (b). The assumed interstellar Fe, Mg and Si reference abundances would not affect the correlation coefficients but the intercept. $R$ is the Pearson correlation coefficient. Also labelled are the Kendall's $\tau$ coefficient and the significance level $p$.
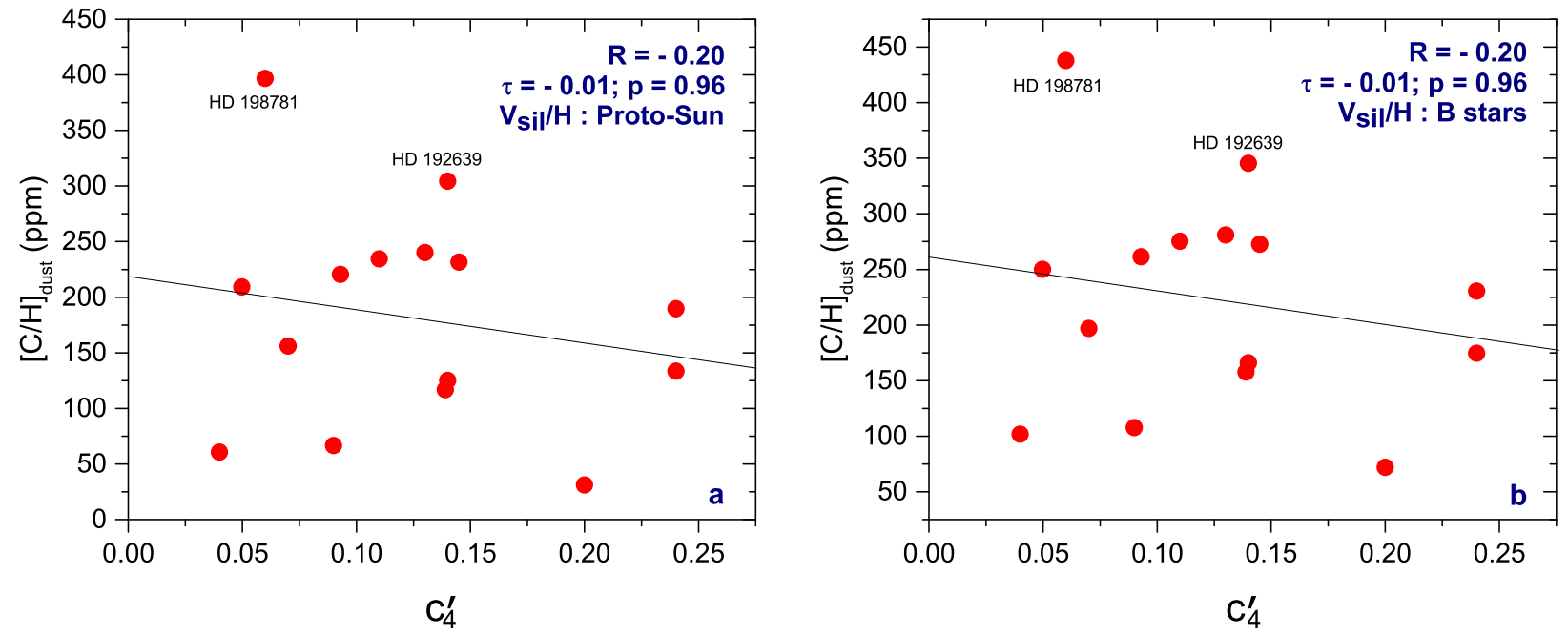

Fig. 5.- Correlation diagrams between the carbon depletion $[\mathrm{C} / \mathrm{H}]_{\text {dust }}$ with the strength of the farUV nonlinear extinction rise $\left(c_{4}^{\prime}\right)$. The carbon depletion is derived from the Kramers-Kronig relation (see eqs.3[14), with the interstellar Fe, Mg and Si abundances taken to be that of the proto-Sun (a) or B stars (b). 

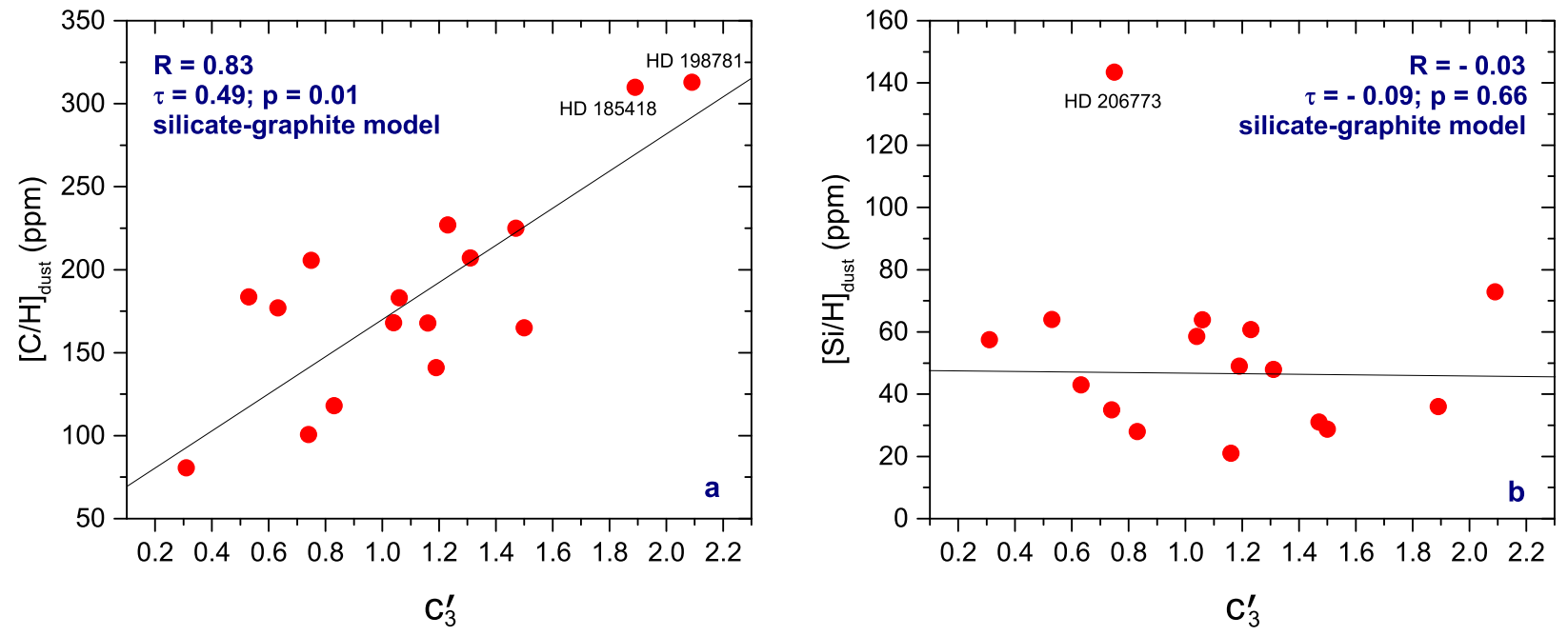

Fig. 6. - Correlation diagrams between the strength of the $2175 \AA$ extinction bump $\left(c_{3}^{\prime}\right)$ with the carbon depletion $[\mathrm{C} / \mathrm{H}]_{\text {dust }}$ (a) and silicon depletion $[\mathrm{Si} / \mathrm{H}]_{\text {dust }}$ (b) derived from fitting the extinction of each sightline with a mixture of silicate dust and graphite dust (see 93 ).
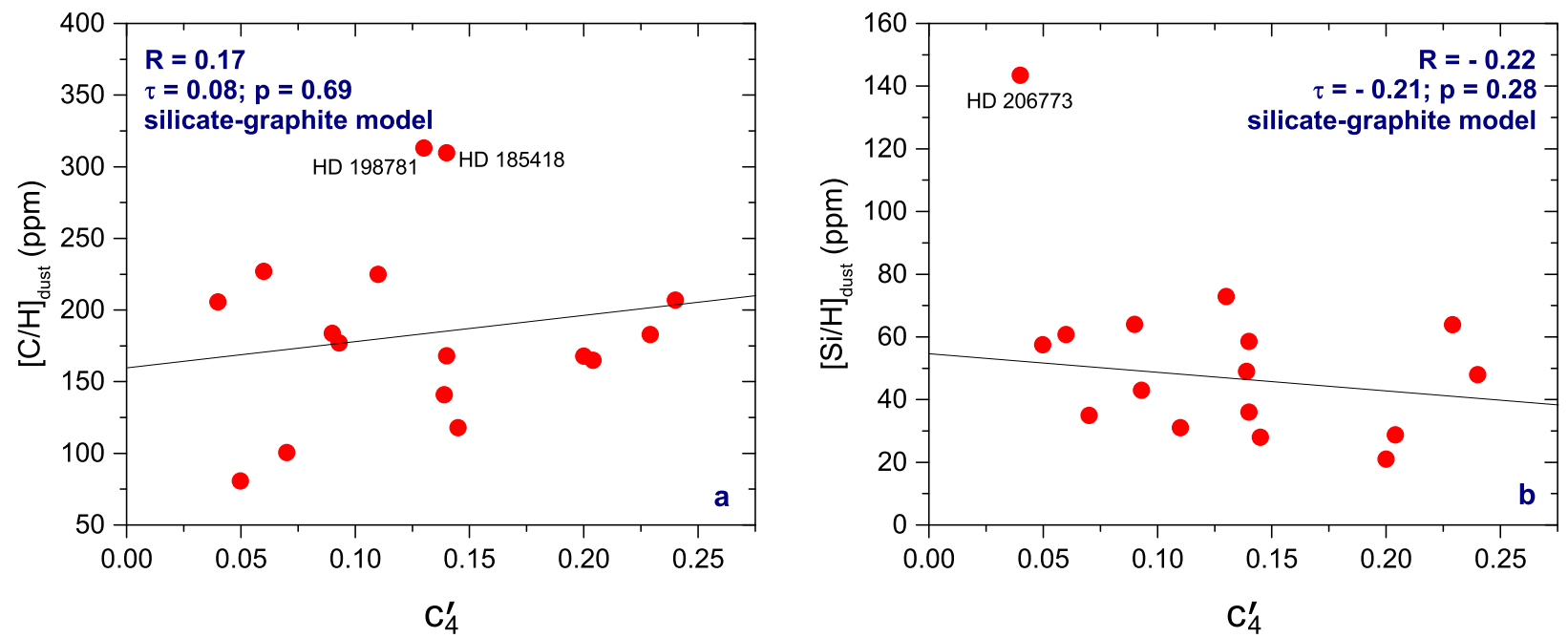

Fig. 7.- Correlation diagrams between the strength of the nonlinear far-UV extinction rise $\left(c_{4}^{\prime}\right)$ with the carbon depletion $[\mathrm{C} / \mathrm{H}]_{\text {dust }}$ (a) and silicon depletion $[\mathrm{Si} / \mathrm{H}]_{\text {dust }}(\mathrm{b})$ derived from fitting the extinction of each sightline with a mixture of silicate dust and graphite dust (see 93 ). 

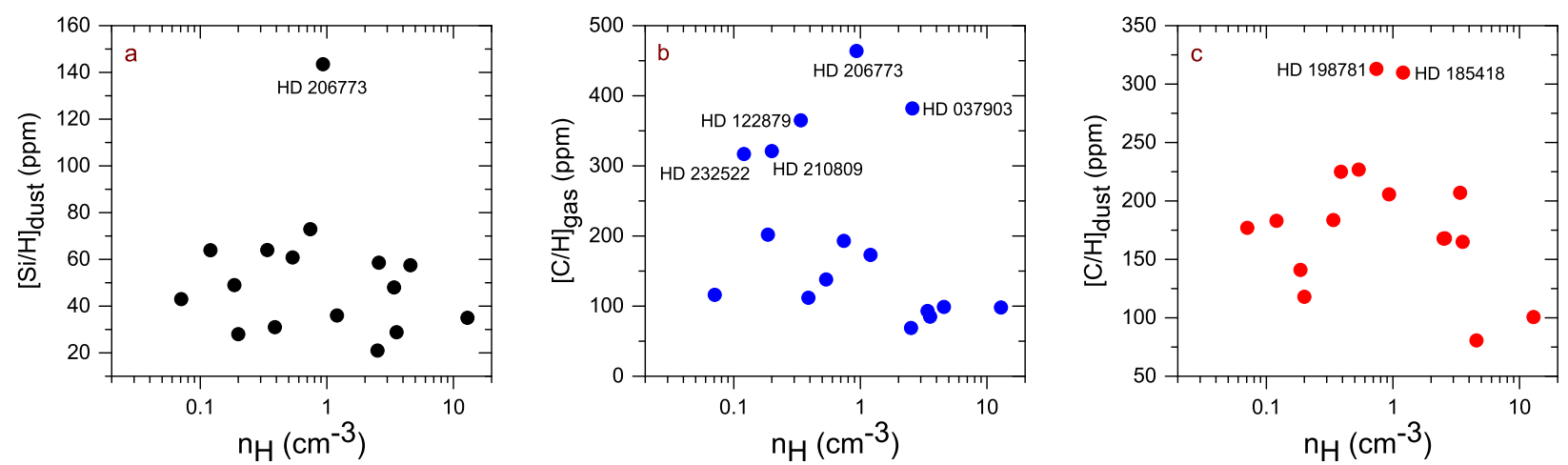

Fig. 8. - Silicon $\left([\mathrm{Si} / \mathrm{H}]_{\text {dust }}\right)$ and carbon $\left([\mathrm{C} / \mathrm{H}]_{\text {dust }}\right)$ depletions as well as gas-phase carbon abundance $\left([\mathrm{C} / \mathrm{H}]_{\text {gas }}\right)$ plotted as a function of the average hydrogen density $\left\langle n_{\mathrm{H}}\right\rangle$. The $[\mathrm{Si} / \mathrm{H}]_{\text {dust }}$ and $[\mathrm{C} / \mathrm{H}]_{\text {dust }}$ depletions are derived from fitting the extinction of each sightline with a mixture of silicate dust and graphite dust (see (3).
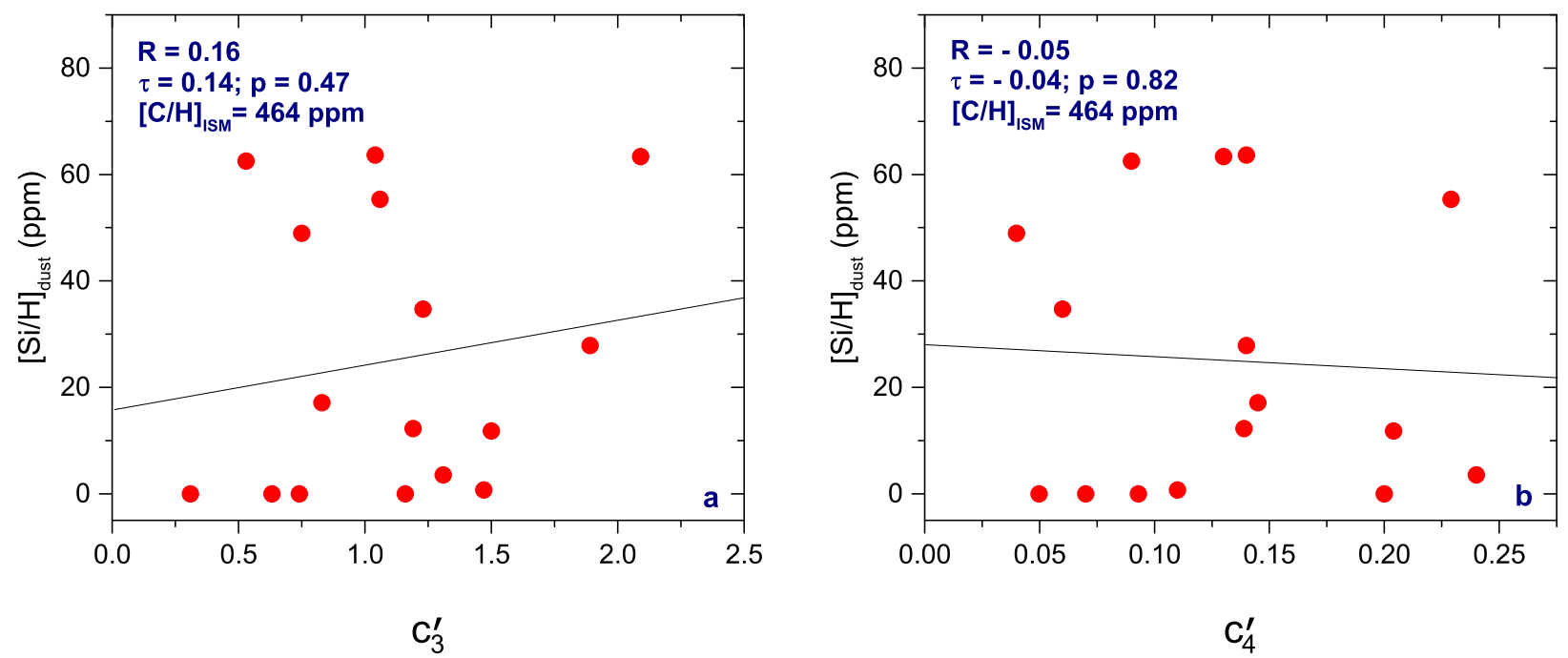

Fig. 9.- Correlation diagrams between the $\mathrm{Si} / \mathrm{H}$ depletion $[\mathrm{Si} / \mathrm{H}]_{\text {dust }}$ with the strength of the extinction bump $\left(c_{3}^{\prime}\right.$; a) and with the far-UV extinction rise $\left(c_{4}^{\prime} ; \mathrm{b}\right)$. The $\mathrm{Si} / \mathrm{H}$ depletion is derived from the KramersKronig relation, with the total $\mathrm{C} / \mathrm{H}$ abundance fixed at $[\mathrm{C} / \mathrm{H}]_{\mathrm{ISM}}=464 \mathrm{ppm}$. 

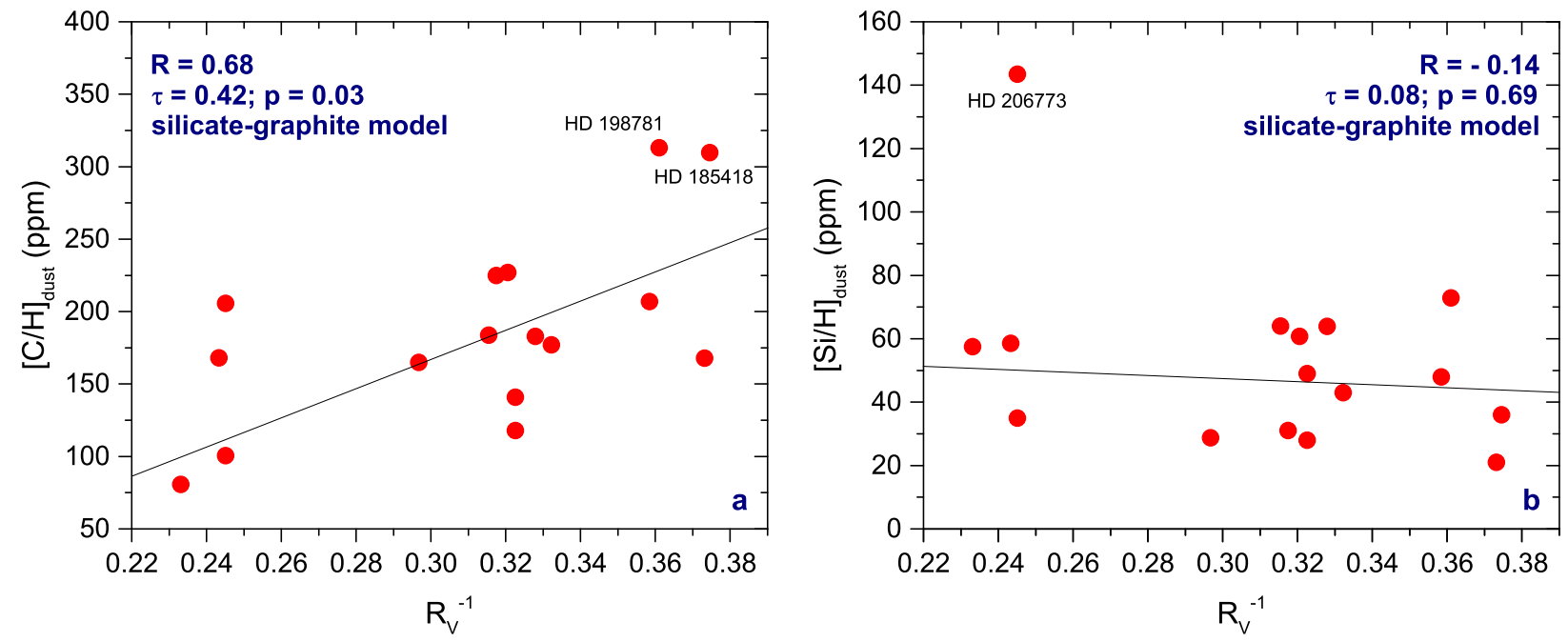

Fig. 10.- Correlation diagrams between $R_{V}^{-1}$ and the carbon depletion $[\mathrm{C} / \mathrm{H}]_{\text {dust }}$ (a) and silicon depletion $[\mathrm{Si} / \mathrm{H}]_{\text {dust }}$ (b) derived from fitting the extinction of each sightline with a mixture of silicate dust and graphite dust (see \$3).
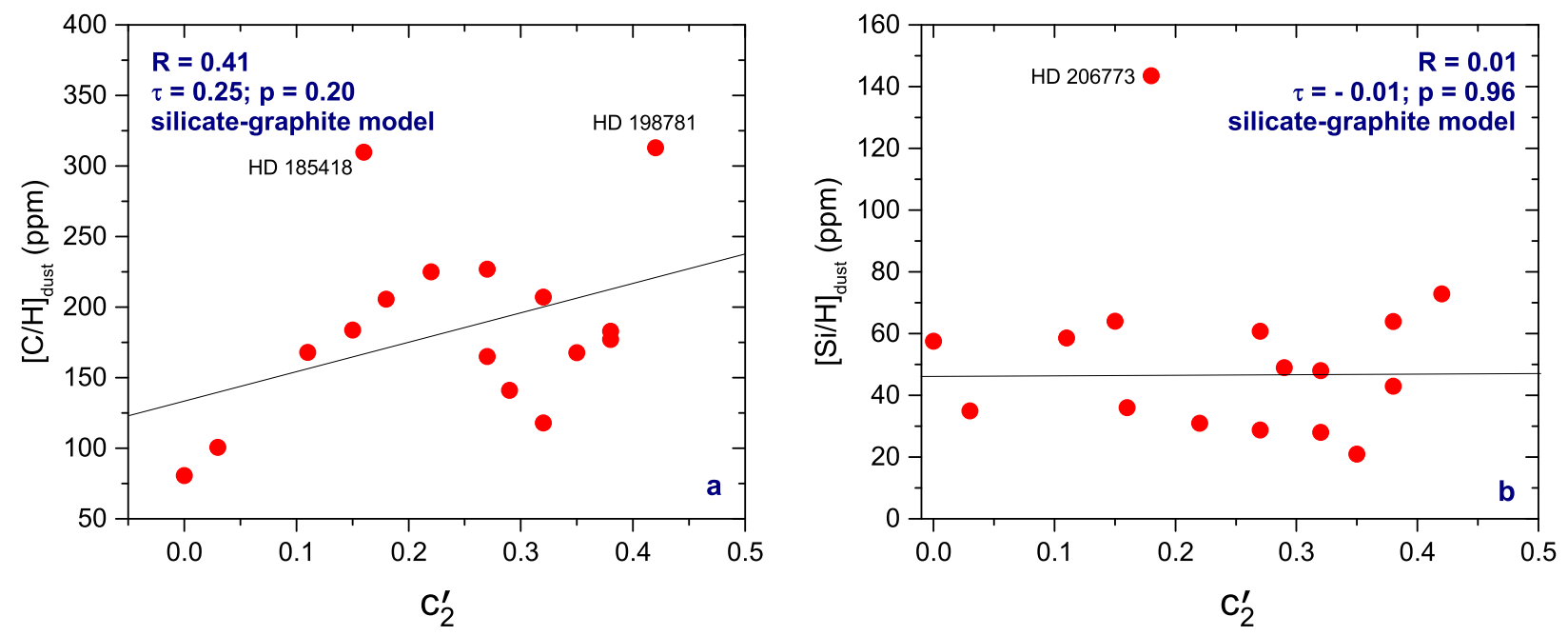

Fig. 11. - Correlation diagrams between the linear background $\left(c_{2}^{\prime}\right)$ with the carbon depletion $[\mathrm{C} / \mathrm{H}]_{\text {dust }}$ (a) and silicon depletion $[\mathrm{Si} / \mathrm{H}]_{\text {dust }}$ (b) derived from fitting the extinction of each sightline with a mixture of silicate dust and graphite dust. 
Table 1. Extinction Parameters and the Carbon Depletion Required to Account for the Observed Extinction

\begin{tabular}{|c|c|c|c|c|c|c|c|c|c|c|c|c|c|c|}
\hline \multirow[t]{2}{*}{ Star } & \multirow{2}{*}{$\begin{array}{c}N_{\mathrm{H}} \\
\left(10^{21} \mathrm{~cm}^{-2} \mathrm{H}\right)\end{array}$} & \multirow{2}{*}{$\begin{array}{c}A_{V} \\
(\mathrm{mag})\end{array}$} & \multirow[t]{2}{*}{$R_{V}$} & \multirow[t]{2}{*}{$c_{1}^{\prime}$} & \multirow[t]{2}{*}{$c_{2}^{\prime}$} & \multirow[t]{2}{*}{$c_{3}^{\prime}$} & \multirow[t]{2}{*}{$c_{4}^{\prime}$} & \multirow{2}{*}{$\begin{array}{c}x_{0} \\
\left(\mu \mathrm{m}^{-1}\right)\end{array}$} & \multirow{2}{*}{$\begin{array}{c}\gamma \\
\left(\mu \mathrm{m}^{-1}\right)\end{array}$} & \multirow{2}{*}{$\begin{array}{c}A_{\mathrm{int}}^{a} \\
\left(\mathrm{mag} \mathrm{cm}^{3} \mathrm{H}^{-1}\right)\end{array}$} & \multicolumn{2}{|l|}{ Proto-Sun ${ }^{b}$} & \multicolumn{2}{|l|}{ B Stars ${ }^{c}$} \\
\hline & & & & & & & & & & & $V_{\mathrm{C}} / \mathrm{H}^{e}$ & {$[\mathrm{C} / \mathrm{H}]_{\mathrm{dust}}^{f}$} & $V_{\mathrm{C}} / \mathrm{H}^{e}$ & {$[\mathrm{C} / \mathrm{H}]_{\text {dust }}^{f}$} \\
\hline HD 027778 & $2.29 \pm 1.20$ & $1.09 \pm 0.03$ & $2.79 \pm 0.38$ & $0.87 \pm 0.71$ & $0.32 \pm 0.01$ & $1.31 \pm 0.10$ & $0.24 \pm 0.01$ & $4.59 \pm 0.04$ & $1.21 \pm 0.03$ & $1.39 \mathrm{E}-25$ & $1.69 \mathrm{E}-27$ & 190 & $2.05 \mathrm{E}-27$ & 231 \\
\hline HD $037061^{d}$ & $5.37 \pm 1.23$ & $2.40 \pm 0.21$ & $4.29 \pm 0.21$ & $1.54 \pm 0.00$ & $0.00 \pm 0.00$ & $0.31 \pm 0.00$ & $0.05 \pm 0.00$ & $4.57 \pm 0.00$ & $0.90 \pm 0.00$ & $1.19 \mathrm{E}-25$ & $1.19 \mathrm{E}-27$ & 134 & $1.55 \mathrm{E}-27$ & 175 \\
\hline HD 037903 & $2.88 \pm 1.15$ & $1.49 \pm 0.03$ & $4.11 \pm 0.43$ & $1.17 \pm 0.06$ & $0.11 \pm 0.01$ & $1.04 \pm 0.09$ & $0.14 \pm 0.01$ & $4.60 \pm 0.04$ & $1.19 \pm 0.03$ & $1.46 \mathrm{E}-25$ & $1.86 \mathrm{E}-27$ & 209 & $2.22 \mathrm{E}-27$ & 250 \\
\hline HD 091824 & $1.44 \pm 1.12$ & $0.60 \pm 0.06$ & $3.10 \pm 0.31$ & $0.74 \pm 0.41$ & $0.29 \pm 0.07$ & $1.19 \pm 0.35$ & $0.14 \pm 0.02$ & $4.62 \pm 0.09$ & $1.00 \pm 0.10$ & $1.16 \mathrm{E}-25$ & $1.11 \mathrm{E}-27$ & 125 & $1.47 \mathrm{E}-27$ & 166 \\
\hline HD $116852^{d}$ & $1.05 \pm 1.20$ & $0.51 \pm 0.12$ & $2.42 \pm 0.37$ & $0.52 \pm 0.25$ & $0.38 \pm 0.10$ & $0.63 \pm 0.17$ & $0.01 \pm 0.01$ & $4.55 \pm 0.04$ & $0.78 \pm 0.07$ & $1.13 \mathrm{E}-25$ & $1.03 \mathrm{E}-27$ & 117 & $1.40 \mathrm{E}-27$ & 158 \\
\hline HD 122879 & $2.19 \pm 1.26$ & $1.41 \pm 0.04$ & $3.17 \pm 0.20$ & $1.15 \pm 0.08$ & $0.15 \pm 0.02$ & $0.53 \pm 0.06$ & $0.09 \pm 0.01$ & $4.57 \pm 0.04$ & $0.74 \pm 0.02$ & $1.50 \mathrm{E}-25$ & $1.96 \mathrm{E}-27$ & 220 & $2.32 \mathrm{E}-27$ & 261 \\
\hline HD 147888 & $5.89 \pm 1.20$ & $1.97 \pm 0.03$ & $4.08 \pm 0.18$ & $1.43 \pm 0.05$ & $0.03 \pm 0.01$ & $0.74 \pm 0.07$ & $0.07 \pm 0.01$ & $4.58 \pm 0.03$ & $0.94 \pm 0.02$ & $9.51 \mathrm{E}-26$ & $5.93 \mathrm{E}-28$ & 66.7 & $9.54 \mathrm{E}-28$ & 108 \\
\hline HD 157857 & $2.75 \pm 1.17$ & $1.37 \pm 0.05$ & $3.15 \pm 0.30$ & $0.78 \pm 0.09$ & $0.22 \pm 0.02$ & $1.47 \pm 0.12$ & $0.11 \pm 0.01$ & $4.54 \pm 0.03$ & $0.96 \pm 0.02$ & $1.27 \mathrm{E}-25$ & $1.39 \mathrm{E}-27$ & 156 & $1.75 \mathrm{E}-27$ & 197 \\
\hline HD 185418 & $2.57 \pm 1.17$ & $1.39 \pm 0.04$ & $2.67 \pm 0.20$ & $1.37 \pm 0.09$ & $0.16 \pm 0.02$ & $1.89 \pm 0.12$ & $0.14 \pm 0.01$ & $4.59 \pm 0.03$ & $1.03 \pm 0.02$ & $1.55 \mathrm{E}-25$ & $2.08 \mathrm{E}-27$ & 234 & $2.45 \mathrm{E}-27$ & 275 \\
\hline HD 192639 & $3.02 \pm 1.17$ & $2.06 \pm 0.04$ & $3.12 \pm 0.16$ & $0.78 \pm 0.05$ & $0.27 \pm 0.01$ & $1.23 \pm 0.07$ & $0.06 \pm 0.01$ & $4.57 \pm 0.02$ & $0.96 \pm 0.02$ & $1.80 \mathrm{E}-25$ & $2.71 \mathrm{E}-27$ & 304 & 3.07E-27 & 345 \\
\hline HD 198781 & $1.41 \pm 1.15$ & $1.03 \pm 0.03$ & $2.77 \pm 0.29$ & $0.44 \pm 0.09$ & $0.42 \pm 0.02$ & $2.09 \pm 0.23$ & $0.13 \pm 0.02$ & $4.69 \pm 0.06$ & $1.36 \pm 0.03$ & $2.13 \mathrm{E}-25$ & $3.53 \mathrm{E}-27$ & 397 & 3.89E-27 & 438 \\
\hline HD $203532^{d}$ & $2.75 \pm 1.17$ & $0.94 \pm 0.11$ & $3.37 \pm 0.24$ & $0.76 \pm 0.16$ & $0.27 \pm 0.03$ & $1.50 \pm 0.25$ & $0.20 \pm 0.04$ & $4.59 \pm 0.01$ & $1.26 \pm 0.04$ & $1.57 \mathrm{E}-25$ & $2.13 \mathrm{E}-27$ & 240 & $2.50 \mathrm{E}-27$ & 281 \\
\hline HD 206773 & $1.78 \pm 1.12$ & $2.16 \pm 0.04$ & $4.08 \pm 0.29$ & $0.84 \pm 0.07$ & $0.18 \pm 0.01$ & $0.75 \pm 0.07$ & $0.04 \pm 0.01$ & $4.57 \pm 0.04$ & $1.04 \pm 0.02$ & $3.34 \mathrm{E}-25$ & $6.52 \mathrm{E}-27$ & 733 & $6.88 \mathrm{E}-27$ & 774 \\
\hline HD 207198 & $4.79 \pm 1.23$ & $1.54 \pm 0.03$ & $2.68 \pm 0.11$ & $0.73 \pm 0.05$ & $0.35 \pm 0.01$ & $1.16 \pm 0.07$ & $0.20 \pm 0.01$ & $4.62 \pm 0.03$ & $1.04 \pm 0.02$ & $9.30 \mathrm{E}-26$ & $5.41 \mathrm{E}-28$ & 60.8 & $9.02 \mathrm{E}-28$ & 102 \\
\hline HD 210809 & $2.14 \pm 1.20$ & $0.98 \pm 0.05$ & $3.10 \pm 0.31$ & $0.60 \pm 0.31$ & $0.32 \pm 0.02$ & $0.83 \pm 0.08$ & $0.15 \pm 0.01$ & $4.60 \pm 0.09$ & $1.00 \pm 0.10$ & $8.24 \mathrm{E}-26$ & $2.77 \mathrm{E}-28$ & 31.1 & $6.38 \mathrm{E}-28$ & 72.1 \\
\hline HD $232522^{d}$ & $1.55 \pm 1.12$ & $0.82 \pm 0.16$ & $3.05 \pm 0.41$ & $0.59 \pm 0.12$ & $0.38 \pm 0.07$ & $1.06 \pm 0.22$ & $0.23 \pm 0.06$ & $4.55 \pm 0.01$ & $0.93 \pm 0.03$ & $1.54 \mathrm{E}-25$ & $2.06 \mathrm{E}-27$ & 232 & $2.42 \mathrm{E}-27$ & 273 \\
\hline
\end{tabular}

${ }^{b}$ The interstellar $\mathrm{Si}, \mathrm{Mg}$ and Fe abundances are taken to be that of proto-Sun (Lodders 2003) for which the total silicate volume per $\mathrm{H}$ atom is $V_{\text {sil }} / \mathrm{H}_{c} \approx 3.17 \times 10^{-27} \mathrm{~cm}^{3} \mathrm{H}^{-1}$.

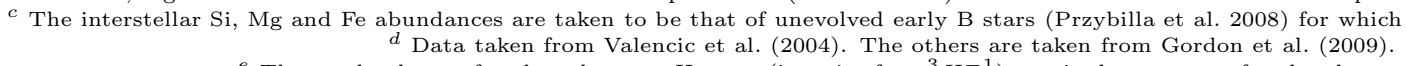

$e$ The total volume of carbon dust per $\mathrm{H}$ atom (in unit of $\mathrm{cm}^{3} \mathrm{H}^{-1}$ ) required to account for the observed extinction.
$f$ The carbon depletion $[\mathrm{C} / \mathrm{H}]_{\text {a }}$ (in unit of $\mathrm{ppm}$ ) required to account for the observed extinction. 
Table 2. Model Parameters for Fitting the UV/Optical/Near-IR Extinction with A Mixture of Silicate/Graphite Dust

\begin{tabular}{cccccccccc}
\hline \hline Star & $\begin{array}{c}A_{V} / N_{\mathrm{H}} \\
\left(10^{-22} \mathrm{mag} \mathrm{cm} \mathrm{H}^{-1}\right)\end{array}$ & $\alpha_{\mathrm{S}}$ & $\begin{array}{c}a_{c, \mathrm{~S}} \\
(\mu \mathrm{m})\end{array}$ & $\alpha_{\mathrm{C}}$ & $\begin{array}{c}a_{c, \mathrm{C}} \\
(\mu \mathrm{m})\end{array}$ & $\chi^{2}$ & $\begin{array}{c}{[\mathrm{C} / \mathrm{H}]_{\text {gas }}} \\
(\mathrm{ppm})\end{array}$ & $\begin{array}{c}{[\mathrm{C} / \mathrm{H}]_{\text {dust }}} \\
(\mathrm{ppm})\end{array}$ & $\begin{array}{c}{[\mathrm{Si} / \mathrm{H}]_{\text {dust }}} \\
(\mathrm{ppm})\end{array}$ \\
\hline HD 027778 & $4.76 \pm 2.50$ & -3.1 & 0.14 & -3.0 & 0.10 & 0.83 & $93 \pm 32$ & 207 & 48.0 \\
HD 037061 & $4.47 \pm 1.10$ & -2.0 & 0.17 & -2.4 & 0.05 & 1.05 & $99 \pm 36$ & 80.7 & 57.5 \\
HD 037903 & $5.17 \pm 2.07$ & -2.7 & 0.23 & -3.4 & 0.20 & 0.47 & $382 \pm 56$ & 168 & 58.6 \\
HD 091824 & $4.17 \pm 3.27$ & -3.1 & 0.24 & -3.8 & 0.25 & 3.19 & $202 \pm 24$ & 141 & 49.0 \\
HD 116852 & $4.86 \pm 5.67$ & -2.7 & 0.08 & -3.4 & 0.15 & 1.54 & $116 \pm 102$ & 177 & 43.0 \\
HD 122879 & $6.44 \pm 3.71$ & -2.7 & 0.18 & -3.4 & 0.10 & 6.19 & $365 \pm 94$ & 184 & 64.0 \\
HD 147888 & $3.34 \pm 0.68$ & -2.1 & 0.16 & -3.5 & 0.20 & 2.28 & $98 \pm 23$ & 101 & 35.0 \\
HD 157857 & $4.98 \pm 2.13$ & -2.5 & 0.08 & -3.9 & 0.40 & 0.14 & $112 \pm 19$ & 225 & 31.0 \\
HD 185418 & $5.05 \pm 2.16$ & -2.0 & 0.06 & -3.7 & 0.30 & 4.14 & $173 \pm 34$ & 310 & 36.0 \\
HD 192639 & $6.82 \pm 2.65$ & -2.6 & 0.10 & -3.5 & 0.10 & 3.16 & $138 \pm 24$ & 227 & 60.8 \\
HD 198781 & $7.30 \pm 5.96$ & -3.0 & 0.10 & -3.1 & 0.10 & 0.12 & $193 \pm 31$ & 313 & 72.9 \\
HD 203532 & $3.42 \pm 1.51$ & -3.3 & 0.21 & -3.2 & 0.15 & 0.87 & $85 \pm 28$ & 165 & 28.8 \\
HD 206773 & $12.1 \pm 7.64$ & -2.7 & 0.20 & -3.1 & 0.05 & 3.40 & $464 \pm 57$ & 206 & 143 \\
HD 207198 & $3.22 \pm 0.83$ & -2.8 & 0.06 & -2.6 & 0.05 & 3.49 & $69 \pm 21$ & 168 & 21.0 \\
HD 210809 & $4.58 \pm 2.58$ & -3.2 & 0.19 & -3.2 & 0.15 & 4.12 & $321 \pm 70$ & 118 & 28.0 \\
HD 232522 & $5.29 \pm 3.96$ & -3.4 & 0.29 & -3.0 & 0.05 & 4.12 & $317 \pm 46$ & 183 & 63.9 \\
\hline
\end{tabular}




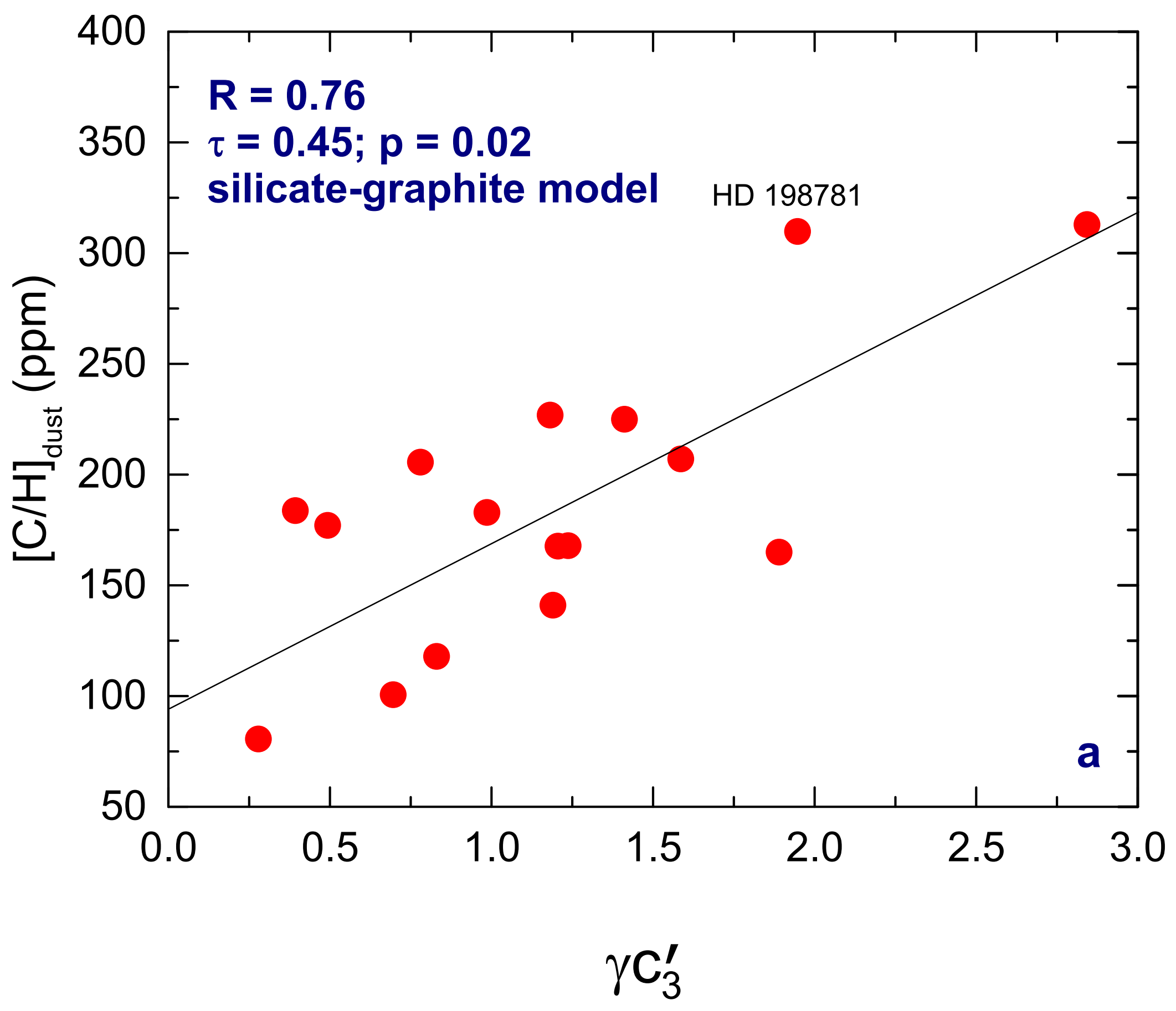

